REPRESENTATION THEORY

An Electronic Journal of the American Mathematical Society

Volume 3, Pages 250-280 (August 31, 1999)

S $1088-4165(99) 00077-1$

\title{
THE INVARIANT POLYNOMIALS ON SIMPLE LIE SUPERALGEBRAS
}

\author{
ALEXANDER SERGEEV
}

\begin{abstract}
Chevalley's theorem states that for any simple finite dimensional Lie algebra $\mathfrak{g}$ : (1) the restriction homomorphism of the algebra of polynomials $S\left(\mathfrak{g}^{*}\right) \longrightarrow S\left(\mathfrak{h}^{*}\right)$ onto the Cartan subalgebra $\mathfrak{h}$ induces an isomorphism $S\left(\mathfrak{g}^{*}\right)^{\mathfrak{g}} \cong S\left(\mathfrak{h}^{*}\right)^{W}$, where $W$ is the Weyl group of $\mathfrak{g} ;(2)$ each $\mathfrak{g}$-invariant polynomial is a linear combination of the polynomials $\operatorname{tr} \rho(x)^{k}$, where $\rho$ is a finite dimensional representation of $\mathfrak{g}$

None of these facts is necessarily true for simple Lie superalgebras. We reformulate Chevalley's theorem as formula $(*)$ below to include Lie superalgebras. Let $\mathfrak{h}$ be the split Cartan subalgebra of $\mathfrak{g}$; let $R=R_{+} \cup R_{-}$be the set of nonzero roots of $\mathfrak{g}$, the union of positive and negative ones. Set $\tilde{R}_{+}=\left\{\alpha \in R_{+} \mid-\alpha \in R_{-}\right\}$. For each root $\alpha \in \tilde{R}_{+}$denote by $\mathfrak{g}(\alpha)$ the Lie superalgebra generated by $\mathfrak{h}$ and the root superspaces $\mathfrak{g}_{\alpha}$ and $\mathfrak{g}_{-\alpha}$. Let the image of $S\left(\mathfrak{g}(\alpha)^{*}\right)^{\mathfrak{g}(\alpha)}$ under the restriction homomorphism $S\left(\mathfrak{g}(\alpha)^{*}\right) \longrightarrow S\left(\mathfrak{h}^{*}\right)$ be denoted by $I^{\alpha}\left(\mathfrak{h}^{*}\right)$ and the image of $S\left(\mathfrak{g}^{*}\right)^{\mathfrak{g}}$ by $I\left(\mathfrak{h}^{*}\right)$. Then
\end{abstract}

$$
I\left(\mathfrak{h}^{*}\right)=\bigcap_{\alpha \in \tilde{R}_{+}} I^{\alpha}\left(\mathfrak{h}^{*}\right) .
$$

Chevalley's theorem for anti-invariant polynomials is also presented.

\section{INTRODUCTION}

All Lie algebras, superalgebras and their representations are assumed to be of finite dimension over $\mathbb{C}$.

0.1. Lie algebras. The algebras of invariant polynomials on semisimple Lie algebras $\mathfrak{g}$ play an important role. In particular, they allow one to describe the structure of the center $Z(\mathfrak{g})$ of the universal enveloping algebra $U(\mathfrak{g})$ and deduce the Weyl character formula for the finite dimensional irreducible representations; the characteristic classes of vector bundles, analogs of Euler's equation of the solid body, etc. are expressed in terms of the invariant polynomials.

If $\mathfrak{g}$ is semisimple, then it possesses an invariant nondegenerate symmetric bilinear form which induces an isomorphism $\mathfrak{g} \simeq \mathfrak{g}^{*}$. This isomorphism commutes with the $\mathfrak{g}$-action and reduces the description of the center to the description of the $\mathfrak{g}$-invariant polynomials on $\mathfrak{g}$, i.e., the elements of $S\left(\mathfrak{g}^{*}\right)^{\mathfrak{g}}$.

The study of the algebras $S\left(\mathfrak{g}^{*}\right)^{\mathfrak{g}}$ has the following important aspects:

1) If $\mathfrak{g}$ is semisimple, then having described $S\left(\mathfrak{g}^{*}\right)^{\mathfrak{g}}$ we simultaneously describe $Z(\mathfrak{g})$ : these algebras are isomorphic.

Received by the editors April 22, 1999 and, in revised form, June 28, 1999.

1991 Mathematics Subject Classification. Primary 17A70; Secondary 17B35, 13A50.

Key words and phrases. Lie superalgebra, invariant theory.

I am thankful to D. Leites for help and support. 
2) Let $\mathfrak{g}$ be semisimple, $\mathfrak{h}$ its Cartan subalgebra, $W$ the Weyl group. Then Chevalley's theorem states that the restriction homomorphism $S\left(\mathfrak{g}^{*}\right) \longrightarrow S\left(\mathfrak{h}^{*}\right)$ onto the Cartan subalgebra induces an isomorphism of the algebra of $\mathfrak{g}$-invariant polynomials on $\mathfrak{g}$ with the algebra of $W$-invariant polynomials on $\mathfrak{h}$ :

$$
S\left(\mathfrak{g}^{*}\right)^{\mathfrak{g}} \cong S\left(\mathfrak{h}^{*}\right)^{W} .
$$

Moreover, each invariant polynomial of degree $k$ is a linear combination of the functions

$$
\operatorname{tr} \rho(x)^{k},
$$

where $\rho$ runs over finite dimensional representations of $\mathfrak{g}$.

3) The algebras of $W$-invariant polynomials are important examples of the algebras of invariant functions; for instance, for $\mathfrak{g}=\mathfrak{s l}(n)$ this leads to the classical invariant theory of symmetric functions.

4) For a semi-simple Lie algebra there exists a canonical homomorphism (called Harish-Chandra homomorphism)

$$
Z(\mathfrak{g}) \longrightarrow S(\mathfrak{h}) \simeq S\left(\mathfrak{h}^{*}\right) .
$$

The image of the center under this homomorphism is the so-called algebra of shifted symmetric functions related with the quantum immanents (that constitute a distinguished basis of $Z(\mathfrak{g}))$, cf. [OO].

0.2. Lie superalgebras. This paper is an attempt to realize part of the above program (1)-(4) for simple Lie superalgebras. Let me point out the main distinctions:

i) not every simple Lie superalgebra possesses a nondegenerate symmetric invariant bilinear form. Even if it does possess one, the form might be odd, while for isomorphism (0.1) we need an even form.

ii) The notion of the Weyl group becomes very involved in the supersetting. Its definition varies depending on the situation; moreover, even for the same problem the description of the analog differs drastically from one type of Lie superalgebras to another one.

iii) not every irreducible $\mathfrak{g}$-module is uniquely determined by its central character (this resembles the case of infinite dimensional modules over classical Lie algebras); hence, the central characters - i.e., the invariant polynomials - provide us with the character formula only for the generic - typical - representations;

iv) for several Lie superalgebras not every invariant polynomial can be represented as a linear combination of supertraces of finite dimensional representations.

Recall that there are several types of simple Lie superalgebras with quite distinct properties:

a) possessing a Cartan matrix (have a reductive even part);

b) with a nondegenerate invariant odd supersymmetric bilinear form (have a reductive even part);

c) with a reductive even part but without a Cartan matrix or a nondegenerate bilinear form;

d) with nonreductive even part.

Here are several distinct definitions of analogs of the Weyl group (ordered historically):

A) as the set over which one performs the summation in the character formula (Bernstein-Leites character formula for atypical representations of $\mathfrak{o s p}(2 \mid 2 n)([\mathrm{L} 1])$ 
and $\mathfrak{s l}(1 \mid n)([\mathrm{BL} 1])$; or its conjectural generalization due to Penkov and Serganova [PS1]);

B) as the group generated by "reflections in simple roots" (Skornyakov, Serganova and Egorov $[\mathrm{E}])$;

C) as the set of neighboring systems of simple roots. The neighboring systems that are related by reflections in even roots can be, as well, considered equivalent under the action of the Weyl group of the even part of $\mathfrak{g}$; the other systems are related by reflections in odd roots whose product is not a priori defined; cf. [E];

D) as the group whose elements number the Schubert supercells (Manin and A. Voronov).

How to define the reflection in the odd root $\alpha$ is not immediately clear. For example, in one of the simplest cases, i.e., for the general matrix algebra $\mathfrak{g l}(m \mid n)$ or its supertraceless subalgebra, we have $(\alpha, \alpha)=0$ for any odd root $\alpha$ whereas in the formula for the reflection one has to divide by this scalar product.

Though Skornyakov, Penkov and Serganova, as well as Egorov, and (in a different framework) Manin suggested several working definitions of the analog of the Weyl group, the very diversity of answers was one of the reasons why in this paper I try to reformulate the Chevalley theorem so as to completely avoid appealing to the notion of the Weyl group and make use of the root decomposition only.

Such formulation is formally applicable to any Lie algebra and Lie superalgebra provided one accordingly modifies the notion of "the root decomposition". The corresponding statement is offered as Conjecture 0.4.3.

The description of the invariants already involves many cases. To make reading easier, the description of invariant polynomials on $\mathfrak{q}$ and its relatives, $\mathfrak{s q}$ and $\mathfrak{p s q}$, will be given elsewhere together with the description of invariant polynomials on the Poisson superalgebra po and its relatives.

0.3. Earlier results. The first to completely describe the invariant polynomials was F. Berezin who did it for a real form of $\mathfrak{g l}(m \mid n)$; see [Be1]. Simultaneously he started to consider the general case with V. Kac but they split and published their results (obtained by distinct methods) separately [Be2] and [K2].

Berezin's proof [Be2] was based on analytical methods; its presentation is not user-friendly. The statements, nevertheless, were a source of inspiration for several researchers. Observe that Berezin only considered Lie superalgebras with root spaces of multiplicity one and with a nondegenerate invariant supersymmetric even bilinear form.

In the addition to the above, Kac [K2] also described a rough structure of the algebra $I(\mathfrak{g})$ of invariant polynomials on the Lie superalgebra $\mathfrak{g}$ of the same class that Berezin considered. Kac's proof is rather lucid.

Berezin and Kac showed that there exists a polynomial $Q$ such that the localization of $I(\mathfrak{g})$ with respect to the multiplicative system generated by $Q$ contains all $W\left(\mathfrak{g}_{\overline{0}}\right)$-invariant polynomials.

In [S1], [S2] and [S3] I described (a bit more explicitly than in [Be1], [Be2] or $[\mathrm{K} 1],[\mathrm{K} 2]$ ) invariant polynomials on Lie superalgebras of series $\mathfrak{g l}, \mathfrak{s l}, \mathfrak{o s p}$ and the exceptional Lie superalgebras as well as on per, $\mathfrak{s p e}, \mathfrak{q}, \mathfrak{v e c t}, \mathfrak{s v e c t}, \widetilde{\mathfrak{s v e c t}}$.

The referee suggested to expand the list or references with some partial earlier results: [K3] (where an interesting method for description of the center of $U(\mathfrak{g})$ for certain Lie superalgebras $\mathfrak{g}$ is given and applied without proof), [Sch] (where the center of $\mathfrak{s p e}(n)$ is described independently of an earlier result of [S1]); [Pe2] and 
[PS2], where there is a discussion of some results of Kac, Sergeev and Scheunert, as well as some applications of their results.

0.4. Main result and conjectures. Let $\mathfrak{g}$ be one of the following series or exceptional finite dimensional Lie superalgebras (in suggestive notations from the review [L2]):

$$
\mathfrak{g l}, \mathfrak{s l}, \mathfrak{p s l}, \mathfrak{o s p}, \mathfrak{p e}, \mathfrak{s p e}, \mathfrak{o s p}_{\alpha}(4 \mid 2) ; \quad \mathfrak{a g}_{2}, \mathfrak{a b}_{3} ; \quad \mathfrak{v e c t}, \mathfrak{s v e c t}, \widetilde{\mathfrak{s v e c t}}
$$

Let $\mathfrak{h}$ be the split Cartan subalgebra of $\mathfrak{g}$; let $R=R_{+} \cup R_{-}$be the set of nonzero roots of $\mathfrak{g}$ divided into subsets of positive and negative ones. Let

$$
\tilde{R}_{+}=\left\{\alpha \in R_{+} \mid-\alpha \in R_{-}\right\} .
$$

For each root $\alpha \in \tilde{R}_{+}$denote by $\mathfrak{g}(\alpha)$ the Lie superalgebra generated by $\mathfrak{h}$ and the root superspaces $\mathfrak{g}_{\alpha}$ and $\mathfrak{g}_{-\alpha}$, i.e. $\mathfrak{g}(\alpha)=\mathfrak{h} \oplus\left(\underset{k \in \mathbb{Z} \backslash 0}{\bigoplus_{k}} \mathfrak{g}_{k \alpha}\right)$. This superalgebra plays an important role in various constructions.

In the cases considered in this paper it is isomorphic to the sum of $\mathfrak{s l}(2)$ or $\mathfrak{o s p}(1 \mid 2)$ or $\mathfrak{s l}(1 \mid 1)$ and $\mathfrak{h}$.

Let the image of $S\left(\mathfrak{g}(\alpha)^{*}\right)^{\mathfrak{g}(\alpha)}$ under the restriction homomorphism $S\left(\mathfrak{g}^{*}\right) \longrightarrow$ $S\left(\mathfrak{h}^{*}\right)$ be denoted by $I^{\alpha}\left(\mathfrak{h}^{*}\right)$ and the image of $S\left(\mathfrak{g}^{*}\right)^{\mathfrak{g}}$ by $I\left(\mathfrak{h}^{*}\right)$.

Theorem (Main Theorem). For the Lie superalgebras (0.1) we have

$$
I\left(\mathfrak{h}^{*}\right)=\bigcap_{\alpha \in \widetilde{R}_{+}} I^{\alpha}\left(\mathfrak{h}^{*}\right) .
$$

If $\tilde{R}_{+}=\emptyset$, then (0.2) should be read as $I\left(\mathfrak{h}^{*}\right)=S\left(\mathfrak{h}^{*}\right)^{\mathfrak{h}}$. This statement is true for semisimple (the direct sum of simple) Lie algebras as well.

Observe that, except for $\mathfrak{g}=\mathfrak{o s p}(1 \mid 2 n)$, the algebra $I\left(\mathfrak{h}^{*}\right)$ is not noetherian whereas the localization of $I\left(\mathfrak{h}^{*}\right)$ with respect to the multiplicative system generated by $Q$ is noetherian.

Define $W(\mathfrak{g})$ to be

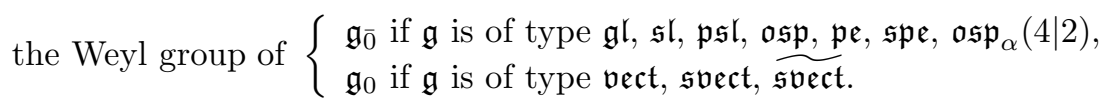

0.4.1. Corollary. Let $Q=\prod_{\alpha \in \widetilde{R}_{\overline{1}}} \alpha$. Then any element of $S\left(\mathfrak{h}^{*}\right)^{W(\mathfrak{g})}$ can be represented in the form $P / Q$, where $P \in I\left(\mathfrak{h}^{*}\right)$.

Problem. Compute the Poincare series of the algebras $I\left(\mathfrak{h}^{*}\right)$. Observe that their description given in section 0.6 depends on the choice of the system of simple roots. It is, perhaps, desirable, to give all such descriptions for all $W(\mathfrak{g})$-inequivalent systems of simple roots; for the list of the latter, found by Serganova, see [LSS].

0.4.2. Conjecture. Equality (0.2) holds for all simple Lie superalgebras (and, moreover, the related "classical" superalgebras, see [L2], i.e., nontrivial central extensions or algebras of differentiations thereof). 
0.4.3. Recall ([PS1]) that if $\mathfrak{g}$ is a nilpotent Lie superalgebra and $V$ a $\mathfrak{g}$-module, then the generalized weight decomposition of $V$ with respect to $\mathfrak{g}$ is a presentation $V=\bigoplus_{\mu \in \mathfrak{g}^{*}} V(\mu)$, where $\mu$ is such that $\mu\left(\left[\mathfrak{g}_{\overline{0}}, \mathfrak{g}_{\overline{0}}\right]\right)=0$ and $V(\mu)$ is the maximal $\mathfrak{g}$-submodule all the irreducible subquotients of which are isomorphic to $\operatorname{Ind}_{\mathfrak{b}}^{\mathfrak{g}}(\mu)$, where $\mathfrak{b}$ is the polarization for $\mu$; see [K1].

Conjecture. Equality (0.2) holds for all Lie algebra and Lie superalgebras provided we consider the generalized weight decompositions.

We suggest that the reader compare our approach with Shander's approach to invariant nonpolynomial functions on Lie superalgebras; cf. [Sh].

0.5. Chevalley's theorem for anti-invariant polynomials. It is well-known ([Bu1]) that there exists a unique extension of the adjoint representation of a Lie algebra $\mathfrak{g}$ to representations in $S(\mathfrak{g})$ and $U(\mathfrak{g})$ and the canonical symmetrization $\omega: S(\mathfrak{g}) \longrightarrow U(\mathfrak{g})$ is a $\mathfrak{g}$-module homomorphism. For Lie superalgebras this supersymmetrization is given by the formula

$$
\omega\left(x_{1}, \ldots, x_{n}\right)=\frac{1}{n} \sum_{\sigma \in \mathfrak{S}_{n}} c(p(x), \sigma) x_{\sigma(1)} \ldots x_{\sigma(n)},
$$

where $p(x)$ is the vector of parities of $x=\left(x_{1}, \ldots, x_{n}\right)$ and $c(p(x), \sigma)$ is defined (see [S1]) as follows. Let $a_{1}, \ldots, a_{n}$ be the elements of the free supercommutative superalgebra. Then

$$
c(p(x), \sigma) a_{1} \ldots a_{n}=a_{\sigma(1)} \ldots a_{\sigma(n)} \text { for any } \sigma \in \mathfrak{S}_{n} .
$$

Consider also the continuation $\tilde{\omega}: S(\mathfrak{g}) \longrightarrow U(\mathfrak{g})$ of the symmetrization $\omega:$ $S\left(\mathfrak{g}_{\overline{0}}\right) \longrightarrow U\left(\mathfrak{g}_{\overline{0}}\right)$, where $S(\mathfrak{g})$ is considered as $\operatorname{Ind}_{\mathfrak{g}_{\overline{0}}}^{\mathfrak{g}}\left(S\left(\mathfrak{g}_{\overline{0}}\right)\right)$ and $U(\mathfrak{g})$ is considered as a $\mathfrak{g}$-module with the action

$$
x * u=(-1)^{p(x)} x u-(-1)^{p(x) p(u)} u x \text { for } x \in \mathfrak{g}, u \in U(\mathfrak{g}) .
$$

Theorem. Let $\mathfrak{g}$ be one of the Lie superalgebras from our list $(0.1), \mathfrak{h}$ its split Cartan subalgebra, $W$ the Weyl group defined as in (0.3) Then the restriction homomorphism $S\left(\mathfrak{g}^{*}\right) \longrightarrow S\left(\mathfrak{h}^{*}\right)$ onto Cartan subalgebra induces an isomorphism

$$
S\left(\mathfrak{g}^{*}\right)^{\mathfrak{g}} \cong S\left(\mathfrak{h}^{*}\right)^{W}
$$

\subsection{Summary: description of the algebra of invariant polynomials.}

0.6.1. $\mathfrak{g}=\mathfrak{g l}(n \mid m)$. Let $\varepsilon_{1}, \ldots, \varepsilon_{n}, \delta_{1}, \ldots, \delta_{m}$ be the weights of the standard (identity) $\mathfrak{g l}(n \mid m)$-module in the standard basis. The Weyl group is $W=\mathfrak{S}_{n} \times \mathfrak{S}_{m}$; it acts on the weights by separately permuting the $\varepsilon_{i}$ and the $\delta_{j}$.

We identify $S\left(\mathfrak{h}^{*}\right)$ with $\mathbb{C}\left[\varepsilon_{1}, \ldots, \varepsilon_{n} ; \delta_{1}, \ldots, \delta_{m}\right]$. Then

$$
I\left(\mathfrak{h}^{*}\right)=\left\{f \in \mathbb{C}\left[\varepsilon_{1}, \ldots, \varepsilon_{n}, \delta_{1}, \ldots, \delta_{m}\right]^{W} \mid \frac{\partial f}{\partial \varepsilon_{i}}+\frac{\partial f}{\partial \delta_{j}} \in\left(\varepsilon_{i}-\delta_{j}\right)\right\} .
$$

This is the algebra of supersymmetric polynomials. For its generators we can take either power series

$$
\Delta_{k}=\sum \varepsilon_{i}^{k}-\sum \delta_{j}^{k}
$$

or the coefficients of powers of $t$ in the power series expansion of the rational function

$$
F(t)=\frac{\prod\left(t-\delta_{j}\right)}{\prod\left(t-\varepsilon_{i}\right)}
$$


Denote $I\left(\mathfrak{h}^{*}\right)$ by $I_{n, m}$.

0.6.2. a) $\mathfrak{g}=\mathfrak{s l}(n \mid m) . I\left(\mathfrak{h}^{*}\right)=I_{n, m} /\left(\varepsilon_{1}+\cdots+\varepsilon_{n}-\delta_{1}-\cdots-\delta_{m}\right)$.

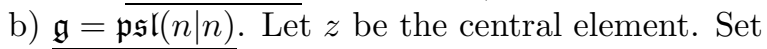

$$
\tilde{I}_{n, n}=\left\{f \in I_{n, n}: D_{z} f \in\left(\varepsilon_{1}+\cdots+\varepsilon_{n}-\delta_{1}-\cdots-\delta_{n}\right)\right\} .
$$

Then $I\left(\mathfrak{h}^{*}\right)=\tilde{I}_{n, n} /\left(\varepsilon_{1}+\cdots+\varepsilon_{n}-\delta_{1}-\cdots-\delta_{n}\right)$.

0.6.3. $\mathfrak{g}=\mathfrak{o s p}(2 m+1 \mid 2 n), m>0$. We identify $S\left(\mathfrak{h}^{*}\right)$ with $\mathbb{C}\left[\varepsilon_{1}, \ldots, \varepsilon_{n}, \delta_{1}, \ldots, \delta_{m}\right]$ generated as algebra by the weights of the standard module. The Weyl group is $W=\left(\mathfrak{S}_{n} \circ \mathbb{Z}_{2}^{n}\right) \times\left(\mathfrak{S}_{m} \circ \mathbb{Z}_{2}^{m}\right)$; it acts on the weights by separately permuting the $\varepsilon_{i}$ and the $\delta_{j}$ and by changing the signs of the weights.

$$
I\left(\mathfrak{h}^{*}\right)=\left\{f \in \mathbb{C}\left[\varepsilon_{1}, \ldots, \varepsilon_{n}, \delta_{1}, \ldots, \delta_{m}\right]^{W} \mid \frac{\partial f}{\partial \varepsilon_{i}} \pm \frac{\partial f}{\partial \delta_{j}} \in\left(\varepsilon_{i} \mp \delta_{j}\right)\right\} .
$$

This is the algebra of supersymmetric polynomials $I_{n, m}$ in $\varepsilon_{1}^{2}, \ldots, \varepsilon_{n}^{2}, \delta_{1}^{2}, \ldots, \delta_{m}^{2}$. For its generators we can take either power series

$$
\Delta_{2 k}=\sum \varepsilon_{i}^{2 k}-\sum \delta_{j}^{2 k}
$$

or the coefficients of powers of $t$ in the power series expansion of the rational function

$$
F(t)=\frac{\prod\left(t^{2}-\varepsilon_{i}^{2}\right)}{\prod\left(t^{2}-\delta_{j}^{2}\right)}
$$

0.6.4. $\underline{\mathfrak{g}=\mathfrak{o} \mathfrak{s p}(1 \mid 2 n)}$. This is a particular case: $F(t)$ is a polynomial.

0.6.5. $\mathfrak{g}=\mathfrak{o s p}(2 m \mid 2 n), m \geq 1$. We identify $S\left(\mathfrak{h}^{*}\right)$ with $\mathbb{C}\left[\varepsilon_{1}, \ldots, \varepsilon_{n}, \delta_{1}, \ldots, \delta_{m}\right]$, as in $0.6 . \overline{3}$.

The Weyl group is $W=\left(\mathfrak{S}_{m} \circ \mathbb{Z}_{2}^{m-1}\right) \times\left(\mathfrak{S}_{n} \circ \mathbb{Z}_{2}^{n}\right)$; it acts on the weights by separately permuting the $\varepsilon_{i}$ and the $\delta_{j}$ and $\mathbb{Z}_{2}^{m-1}$ changes the signs of the weights $\varepsilon_{1} \rightarrow \theta_{i} \varepsilon_{i}$, where $\theta_{i}= \pm 1$ and $\prod \theta_{i}=1$. In this case

$$
I\left(\mathfrak{h}^{*}\right)=\left\{f \in \mathbb{C}\left[\varepsilon_{1}, \ldots, \varepsilon_{m}, \delta_{1}, \ldots, \delta_{n}\right]^{W} \mid \frac{\partial f}{\partial \varepsilon_{i}} \pm \frac{\partial f}{\partial \delta_{j}} \in\left(\varepsilon_{i} \mp \delta_{j}\right)\right\} .
$$

Any element of $I\left(\mathfrak{h}^{*}\right)$ can be expressed in the form

$$
f=f_{0}+\varepsilon_{1} \ldots \varepsilon_{m} \prod_{i, j}\left(\varepsilon_{i}^{2}-\delta_{j}^{2}\right) \cdot f_{1},
$$

where $f_{0} \in I_{n, m}\left(\varepsilon_{1}^{2}, \ldots, \varepsilon_{n}^{2}, \delta_{1}^{2}, \ldots, \delta_{m}^{2}\right)$ and can be expressed via the coefficients of the powers of $t$ in the power series expansion of the rational function

$$
F(t)=\frac{\prod\left(t^{2}-\delta_{j}^{2}\right)}{\prod\left(t^{2}-\varepsilon_{i}^{2}\right)}
$$

and where $f_{1} \in \mathbb{C}\left[\varepsilon_{1}, \ldots, \varepsilon_{n}, \delta_{1}, \ldots, \delta_{m}\right]^{W}$. 
0.6.6. $\mathfrak{g}=\mathfrak{o s p} \mathfrak{p}_{\alpha}(4 \mid 2)$. We identify $S\left(\mathfrak{h}^{*}\right)$ with the algebra $\mathbb{C}\left[\varepsilon_{1}, \varepsilon_{2}, \varepsilon_{3}\right]$.

The Weyl group is $W=\mathbb{Z}_{2} \times \mathbb{Z}_{2} \times \mathbb{Z}_{2}$; it acts on the weights by changing their signs. Set

$$
\lambda_{1}=-(1+\alpha), \quad \lambda_{2}=1, \quad \lambda_{3}=\alpha .
$$

In this case for $\theta_{i}= \pm 1$ we have

$$
\begin{aligned}
I\left(\mathfrak{h}^{*}\right)=\left\{f \in \mathbb{C}\left[\varepsilon_{1}, \varepsilon_{2}, \varepsilon_{3}\right]^{W} \mid \theta_{1} \lambda_{1} \frac{\partial f}{\partial \varepsilon_{1}}\right. & +\theta_{2} \lambda_{2} \frac{\partial f}{\partial \varepsilon_{2}}+\theta_{3} \lambda_{3} \frac{\partial f}{\partial \varepsilon_{3}} \\
& \left.\in\left(\theta_{1} \varepsilon_{1}+\theta_{2} \varepsilon_{2}+\theta_{3} \varepsilon_{3}\right)\right\} .
\end{aligned}
$$

Any element of $I\left(\mathfrak{h}^{*}\right)$ can be expressed in the form

$$
f=f_{0}+\prod\left(\varepsilon_{1} \pm \varepsilon_{2} \pm \varepsilon_{3}\right) \cdot f_{1},
$$

where $f_{0} \in \mathbb{C}\left[\frac{1}{\lambda_{1}} \varepsilon_{1}^{2}+\frac{1}{\lambda_{2}} \varepsilon_{2}^{2}+\frac{1}{\lambda_{3}} \varepsilon_{3}^{2}\right]$ and $f_{1} \in \mathbb{C}\left[\varepsilon_{1}^{2}, \varepsilon_{2}^{2}, \varepsilon_{3}^{2}\right]$.

0.6.7. $\mathfrak{g}=\mathfrak{a g}_{2}$. We identify $S\left(\mathfrak{h}^{*}\right)$ with the algebra $\mathbb{C}\left[\varepsilon_{1}, \varepsilon_{2}, \varepsilon_{3}, \delta\right] /\left(\varepsilon_{1}+\varepsilon_{2}+\varepsilon_{3}\right)$.

The Weyl group $W=\left(\mathfrak{S}_{3} \circ \mathbb{Z}_{2}\right) \times \mathbb{Z}_{2} ; \mathfrak{S}_{3} \circ \mathbb{Z}_{2}$ acts on the $\varepsilon_{i}$ by permutations and simultaneously changes their signs; the second factor, $\mathbb{Z}_{2}$, changes the sign of the $\delta$. In this case

$$
I\left(\mathfrak{h}^{*}\right)=\left\{f \in S\left(\mathfrak{h}^{*}\right)^{W} \mid \frac{\partial f}{\partial \varepsilon_{1}}+\frac{\partial f}{\partial \varepsilon_{2}}+2 \frac{\partial f}{\partial \delta} \in\left(\delta-\varepsilon_{1}-\varepsilon_{2}\right)\right\} .
$$

Any element of $I\left(\mathfrak{h}^{*}\right)$ can be expressed in the form

$$
f=f_{0}+\prod_{1 \leq i \leq 3}\left(\delta^{2}-\varepsilon_{i}^{2}\right) \cdot f_{1},
$$

where $f_{0} \in \mathbb{C}\left[3 \delta^{2}-2\left(\varepsilon_{1}^{2}+\varepsilon_{2}^{2}+\varepsilon_{3}^{2}\right)\right]$ and $f_{1} \in S\left(\mathfrak{h}^{*}\right)^{W}$.

0.6.8. $\mathfrak{g}=\mathfrak{a b}_{3}$. We identify $S\left(\mathfrak{h}^{*}\right)$ with $\mathbb{C}\left[\varepsilon_{1}, \varepsilon_{2}, \varepsilon_{3}, \delta\right]$.

The Weyl group $W=\left(\mathfrak{S}_{3} \circ \mathbb{Z}_{2}^{3}\right) \times \mathbb{Z}_{2} ; \mathfrak{S}_{3} \circ \mathbb{Z}_{2}^{3}$ acts on the $\varepsilon_{i}$ by permutations and changes their signs; the second factor, $\mathbb{Z}_{2}$, changes the sign of the $\delta$. In this case

$$
I\left(\mathfrak{h}^{*}\right)=\left\{f \in S\left(\mathfrak{h}^{*}\right)^{W} \mid \frac{\partial f}{\partial \varepsilon_{1}}+\frac{\partial f}{\partial \varepsilon_{2}}+\frac{\partial f}{\partial \varepsilon_{3}}-3 \frac{\partial f}{\partial \delta} \in\left(\delta+\varepsilon_{1}+\varepsilon_{2}+\varepsilon_{3}\right)\right\} .
$$

Any element of $I\left(\mathfrak{h}^{*}\right)$ can be expressed in the form

$$
f=f_{0}+\prod\left(\delta \pm \varepsilon_{1} \pm \varepsilon_{2} \pm \varepsilon_{3}\right) \cdot f_{1},
$$

where $f_{0} \in \mathbb{C}\left[L_{2}, L_{6}\right], f_{1} \in S\left(\mathfrak{h}^{*}\right)^{W}$ and

$$
\begin{aligned}
L_{2} & =3\left(\varepsilon_{1}^{2}+\varepsilon_{2}^{2}+\varepsilon_{3}^{2}\right)-\delta^{2}, \\
L_{6} & =\delta^{6}+\varepsilon_{1}^{6}+\varepsilon_{2}^{6}+\varepsilon_{3}^{6} \\
& +\left(\varepsilon_{1}-\varepsilon_{2}\right)^{6}+\left(\varepsilon_{1}-\varepsilon_{3}\right)^{6}+\left(\varepsilon_{2}-\varepsilon_{3}\right)^{6}+\left(\varepsilon_{1}+\varepsilon_{2}\right)^{6} \\
& +\left(\varepsilon_{1}+\varepsilon_{3}\right)^{6}+\left(\varepsilon_{2}+\varepsilon_{3}\right)^{6}-\frac{1}{64}\left(\sum\left(\delta \pm \varepsilon_{1} \pm \varepsilon_{2} \pm \varepsilon_{3}\right)^{6}\right) .
\end{aligned}
$$


0.6.9. $\mathfrak{g}=\mathfrak{p} \mathfrak{e}(n)$. We identify $S\left(\mathfrak{h}^{*}\right)$ with the algebra $\mathbb{C}\left[\varepsilon_{1}, \ldots, \varepsilon_{n}\right]$ generated by the weights of the standard $\mathfrak{g}$-module.

The Weyl group $W=\mathfrak{S}_{n}$ acts on the $\varepsilon_{i}$ by permutations. In this case

$$
I\left(\mathfrak{h}^{*}\right)=\left\{f \in S\left(\mathfrak{h}^{*}\right)^{W} \mid \frac{\partial f}{\partial \varepsilon_{i}}-\frac{\partial f}{\partial \varepsilon_{j}} \in\left(\varepsilon_{i}+\varepsilon_{j}\right)\right\} .
$$

This is the algebra of projective Schur functions. For a system of its generators we can take either the sums of powers

$$
\Delta_{2 k+1}=\sum x_{i}^{2 k+1}
$$

or the coefficients of the rational function

$$
F(t)=\frac{\prod\left(t+\varepsilon_{i}\right)}{\prod\left(t-\varepsilon_{i}\right)} .
$$

Set $I_{n}=I\left(\mathfrak{h}^{*}\right)$.

0.6.10. $\mathfrak{g}=\mathfrak{s p e}(n)$. Then $I\left(\mathfrak{h}^{*}\right)=I_{n} /\left(\varepsilon_{1}+\cdots+\varepsilon_{n}\right)$.

0.6.11. $\mathfrak{g}=\operatorname{vect}(0 \mid n)$. We identify $S\left(\mathfrak{h}^{*}\right)$ with the algebra $\mathbb{C}\left[\varepsilon_{1}, \ldots, \varepsilon_{n}\right]$ generated by the weights of the $\mathfrak{g}_{0}$-module $\mathfrak{g}_{-1}$.

The Weyl group $W=\mathfrak{S}_{n}$ acts on the $\varepsilon_{i}$ by permutations.

In this case

$$
I\left(\mathfrak{h}^{*}\right)=\left\{f \in S\left(\mathfrak{h}^{*}\right)^{W} \mid \frac{\partial f}{\partial \varepsilon_{i}} \in\left(\varepsilon_{j}\right) \text { for any } i \neq j\right\} .
$$

Each element of $I\left(\mathfrak{h}^{*}\right)$ is of the form

$$
f=c+\varepsilon_{1} \ldots \varepsilon_{n} g, \text { where } c \in \mathbb{C}, g \in S\left(\mathfrak{h}^{*}\right)^{W} .
$$

Set $J_{n}=I\left(\mathfrak{h}^{*}\right)$.

0.6.12. a) $\mathfrak{g}=\operatorname{svect}(0 \mid n)$. Then $I\left(\mathfrak{h}^{*}\right) \cong J_{n} /\left(\varepsilon_{1}+\cdots+\varepsilon_{n}\right)$.

b) $\mathfrak{g}=\widetilde{\mathfrak{s v e c t}}(0 \mid n)$. Then the algebra of invariant polynomials is the same as in the non-deformed case, i.e., $I\left(\mathfrak{h}^{*}\right) \cong J_{n} /\left(\varepsilon_{1}+\cdots+\varepsilon_{2 n}\right)$.

\section{Preliminaries}

1.1. Proposition $([\mathrm{S} 2])$. Let $\mathfrak{g}$ be a finite dimensional Lie superalgebra, $V$ a finite dimensional $\mathfrak{g}$-module, $L \subset V$ a subsuperspace and $w_{0} \in W_{\overline{0}}$ an element such that the map $\mathfrak{g} \times W \longrightarrow V$ given by the formula

$$
x, w \mapsto x w_{0}+w
$$

is surjective. Then the restriction map $S\left(V^{*}\right)^{\mathfrak{g}} \longrightarrow S\left(W^{*}\right)$ is injective.

Proof. For notations see the Appendix. Let $\rho: \mathfrak{g}_{\Lambda} \longrightarrow \mathfrak{g l}\left(V_{\Lambda}\right)$ be a homomorphism. Let $G_{\Lambda}$ be a connected and simply connected Lie group corresponding to $\mathfrak{g}_{\Lambda}$. By the Lie theory (see $[\mathrm{OV}]$ ) there exists a unique homomorphism $\pi: G_{\Lambda} \longrightarrow G L\left(V_{\Lambda}\right)$ such that the derivative of $\pi$ at the unit is equal to $\rho$. Consider the manifold morphism

$$
\Phi: G_{\Lambda} \times L_{\Lambda} \longrightarrow V_{\Lambda}, \quad \Phi(g, w)=g w .
$$

It is easy to calculate the derivative of this map. It is equal to

$$
(D \Phi)\left(1, w_{0}\right)(\tilde{x}, \tilde{w})=\rho(\tilde{x}) w_{0}+\tilde{w} .
$$


Let $f \in S\left(V^{*}\right)^{\mathfrak{g}}$ and $i(f)=0$. Then $\left.\theta(f)\right|_{L_{\Lambda}}=0$. By Lemma A.6 $\theta(f) \in$ $\Lambda \otimes S\left(V_{\Lambda}^{*}\right)$ is $\mathfrak{g}_{\Lambda}$-invariant. By Corollary 3 of Proposition 3.6.13 from [Bu2] $\theta(f)$ is invariant with respect to the natural $G_{\Lambda}$-action on $\Lambda \otimes S\left(V_{\Lambda}^{*}\right)$.

Consider $\theta(f)$ as the usual polynomial mapping $V_{\Lambda} \longrightarrow \Lambda$. It follows from $(*)$ that $\Phi$ is submersive at $\left(1, w_{0}\right)$. Therefore, the image of $\Phi$ contains an open neighborhood of $w_{0}$ in $V_{\Lambda}$. Hence, $G_{\Lambda} L_{\Lambda}$ contains an open neighborhood of $w_{0}$; hence, $\theta(f)=0$. Lemma A.6 shows that $f=0$.

Corollary. If $\mathfrak{g}$ is a finite dimensional Lie superalgebra, $\mathfrak{h}$ its Cartan subalgebra, then the restriction map $S\left(\mathfrak{g}^{*}\right)^{\mathfrak{g}} \longrightarrow S\left(\mathfrak{h}^{*}\right)$ is injective.

Proof. It suffices to demonstrate that the map $\mathfrak{g} \times \mathfrak{h} \longrightarrow \mathfrak{g}$ given by the formula

$$
x, h \mapsto x h_{0}+h
$$

is surjective.

Let $\mathfrak{g}=\bigoplus_{\alpha \in R} \mathfrak{g}(\alpha)$ be the generalized weight decomposition of $\mathfrak{g}$ relative to $\mathfrak{h}$; see section 0.4.3.

Select $h_{0} \in \mathfrak{h}_{\overline{0}}$ such that $\alpha\left(h_{0}\right) \neq 0$ for all $\alpha \in R$. Then the kernel of the above map consists of the pairs $(x, h)$ such that $x h_{0}+h=0$. Since $\alpha\left(h_{0}\right) \neq 0$ for all $\alpha \in R$, it follows that $x \in \mathfrak{h}$ and the dimension of the kernel is equal to that of $\mathfrak{h}$. Hence, the map $\mathfrak{g} \times \mathfrak{h} \longrightarrow \mathfrak{g}$ is surjective.

1.2. Proposition. Let $\mathfrak{g}$ be a finite dimensional Lie superalgebra such that the Lie algebra $\mathfrak{g}_{\overline{0}}=\mathfrak{h}$ is commutative; $\mathfrak{g}_{\overline{1}}=\operatorname{Span}(u, v)$ and the following relations hold:

$$
\begin{aligned}
& {[h, v]=-\alpha(h) v,[h, u]=\alpha(h) u \text { for any } h \in \mathfrak{h} \text { and } \alpha \in \mathfrak{h}^{*} ;} \\
& {[u, v]=h_{\alpha} \text { and } \alpha\left(h_{\alpha}\right)=0 .}
\end{aligned}
$$

Let

$$
I^{\alpha}\left(\mathfrak{h}^{*}\right)=\left\{f \in S\left(\mathfrak{h}^{*}\right) \mid D_{h_{\alpha}} f \in(\alpha)\right\},
$$

where $D_{h_{\alpha}} f$ is the derivative of $f$ in the direction of $h_{\alpha}$.

Then the restriction homomorphism $S\left(\mathfrak{g}^{*}\right) \longrightarrow S\left(\mathfrak{h}^{*}\right)$ induces an isomorphism $S\left(\mathfrak{g}^{*}\right)^{\mathfrak{g}} \cong I^{\alpha}\left(\mathfrak{h}^{*}\right)$.

Proof. Since $\mathfrak{h}$ is the Cartan subalgebra, the restriction homomorphism is injective by Proposition 1.1. Let us prove that its image coincides with $I^{\alpha}\left(\mathfrak{h}^{*}\right)$.

Let $F \in S\left(\mathfrak{g}^{*}\right)^{\mathfrak{g}}$. By $\mathfrak{h}$-invariance, $F$ should be of the form

$$
F=f+g v^{*} u^{*} \text {, where } v^{*} \text { and } u^{*} \text { is the left dual basis to } v \text { and } u \text {. }
$$

It is easy to verify that in $S\left(\mathfrak{g}^{*}\right)$ the following relations hold:

$$
\begin{aligned}
& v \cdot l=-l\left(h_{\alpha}\right) \cdot u^{*}, u \cdot l=-l\left(h_{\alpha}\right) \cdot v^{*}, \text { for } l \in \mathfrak{h}^{*}, \\
& v \cdot v^{*}=\alpha ; v \cdot u^{*}=0 ; u \cdot u^{*}=-\alpha ; u \cdot v^{*}=0 .
\end{aligned}
$$

Therefore, $v \cdot F=-D_{h_{\alpha}} f \cdot u^{*}+\alpha g u^{*}=0$. Hence, $D_{h_{\alpha}} f=\alpha g$ and $f \in I^{\alpha}\left(\mathfrak{h}^{*}\right)$.

Conversely, if $f \in I^{\alpha}\left(\mathfrak{h}^{*}\right)$, then it is easy to verify that

$$
F=f+\frac{1}{\alpha} D_{h_{\alpha}} f v^{*} u^{*} \in S\left(\mathfrak{g}^{*}\right)^{\mathfrak{g}} .
$$


1.3. Proposition. Let $\mathfrak{g}$ be a finite dimensional Lie superalgebra such that the Lie algebra $\mathfrak{g}_{\overline{0}}=\mathfrak{h}$ is commutative; $\mathfrak{g}_{\overline{1}}=\operatorname{Span}\left(v_{1}, u_{1}, v_{2}, u_{2}\right)$ and the following relations hold:

$$
\begin{aligned}
& {\left[h, v_{i}\right]=-\alpha(h) v_{i},\left[h, u_{i}\right]=\alpha(h) u_{i} \text { for any } h \in \mathfrak{h}, i=1,2 \text { and } \alpha \in \mathfrak{h}^{*} ;} \\
& {\left[u_{i}, u_{j}\right]=\left[v_{i}, v_{j}\right]=0 ;} \\
& \left.\left[u_{i}, v_{j}\right]=0 \text { for } i \neq j ;\left[u_{i}, v_{i}\right]=h_{i} \text { (it is possible that } h_{1}=h_{2}\right) ; \\
& \alpha\left(\left[\mathfrak{g}_{\overline{1}}, \mathfrak{g}_{\overline{1}}\right]\right)=0 .
\end{aligned}
$$

Let

$$
I^{\alpha}\left(\mathfrak{h}^{*}\right)=\left\{f \in S\left(\mathfrak{h}^{*}\right) \mid D_{h_{1}} f \in(\alpha), D_{h_{2}} f \in(\alpha), D_{h_{1}} D_{h_{2}} f \in\left(\alpha^{2}\right)\right\},
$$

where $D_{h} f$ is the derivative of $f$ in the direction of $h$.

Then the restriction homomorphism $S\left(\mathfrak{g}^{*}\right) \longrightarrow S\left(\mathfrak{h}^{*}\right)$ induces an isomorphism $S\left(\mathfrak{g}^{*}\right)^{\mathfrak{g}} \cong I^{\alpha}\left(\mathfrak{h}^{*}\right)$.

Proof. As in the proof of Proposition 1.2, let $F \in S\left(\mathfrak{g}^{*}\right)^{\mathfrak{g}}$. By $\mathfrak{h}$-invariance, $F$ should be of the form

$$
F=f+g v_{1}^{*} u_{1}^{*}+s v_{2}^{*} u_{2}^{*}+r v_{1}^{*} u_{1}^{*} v_{2}^{*} u_{2}^{*},
$$

where the $v_{i}^{*}$ and $u_{i}^{*}$ constitute the left dual basis to the $v_{i}, u_{i}$.

The condition $v_{1} F=0$ implies that

$$
D_{h_{1}} f-\alpha g=0, \quad D_{h_{1}} s-\alpha r=0,
$$

and similarly

$$
D_{h_{2}} f-\alpha s=0, D_{h_{2}} s-\alpha r=0 .
$$

These conditions imply that

$$
D_{h_{1}} f \in(\alpha), \quad D_{h_{2}} f \in(\alpha), \quad D_{h_{1}} D_{h_{2}} f \in\left(\alpha^{2}\right) .
$$

Conversely, if these conditions hold, then by setting

$$
F=f+\frac{1}{\alpha} D_{h_{1}} f v_{1}^{*} u_{1}^{*}+\frac{1}{\alpha} D_{h_{2}} f v_{2}^{*} u_{2}^{*}+\frac{1}{\alpha^{2}} D_{h_{1}} D_{h_{2}} f v_{1}^{*} u_{1}^{*} v_{2}^{*} u_{2}^{*}
$$

we get an element from $S\left(\mathfrak{g}^{*}\right)^{\mathfrak{g}}$ whose restriction on $\mathfrak{h}^{*}$ is equal to $f$.

Remark. Under the conditions of Proposition 1.3, if $h_{1}=h_{2}$, then not every element from $S\left(\mathfrak{g}^{*}\right)^{\mathfrak{g}}$ can be obtained as a linear combination of invariant polynomials of the form $\operatorname{str}\left(\rho(x)^{k}\right)$, where $\rho$ is any finite dimensional representation of $\mathfrak{g}$.

Indeed, let $h_{1}=h_{2}=h$. Then there are two types of representations of $\mathfrak{g}$ :

1) the 1-dimensional ones, determined by linear forms $\lambda \in \mathfrak{h}^{*}$ such that $\lambda(h)=0$ and

2) (4,4)-dimensional ones for which $\lambda(h) \neq 0$; these representations are of the form

$$
T_{\lambda}=\operatorname{ind}_{\mathfrak{h} \oplus \operatorname{Span}\left(u_{1}, u_{2}\right)}^{\mathfrak{g}}(\lambda) .
$$

The character of a representation of the first type is equal (up to $\varepsilon$ ) to $e^{\lambda}$; these characters generate a subalgebra of invariants isomorphic to $\mathbb{C}\left[\mathfrak{h}^{\perp}\right]$, where

$$
\mathfrak{h}^{\perp}=\left\{\lambda \in \mathfrak{h}^{*} \mid \lambda(h)=0\right\} .
$$

Further, it is not difficult to verify that each homogeneous component of the supercharacter in the sense of Kac considered as a formal power series is an invariant polynomial. (Recall that $\operatorname{sch} V$ is a linear functional on $U(\mathfrak{g})$ defined $\operatorname{as} \operatorname{sch} V(u)=$ 
$\operatorname{str}_{V}(u)$; here we identify $U(\mathfrak{g})^{*}$ with the algebra of formal power series.) Actually, this component is $\operatorname{str}\left(\rho(x)^{k}\right)$, where $\rho$ is the corresponding representation of $\mathfrak{g}$.

Therefore, the homogeneous components of supercharacters of $T_{\lambda}$ are invariant polynomials. But the supercharacter of $T_{\lambda}$ is equal to $\left(1-e^{-\alpha}\right)^{2} e^{\lambda}$ and the linear span of such supercharacters is equal to $\alpha^{2} \cdot \varphi$, where $\varphi \in \mathbb{C}\left[\mathfrak{h}^{*}\right]$. Thus, the general form of the restriction of an invariant polynomial equal to the linear combination of supertraces is of the form

$$
\psi+\alpha^{2} \cdot \varphi, \text { where } \varphi \in \mathbb{C}\left[\mathfrak{h}^{*}\right], \psi \in \mathbb{C}\left[\mathfrak{h}^{\perp}\right] .
$$

On the other hand, by Proposition 1.3 the element $\alpha \cdot l$, where $l \in \mathfrak{h}^{*}$ and $l(h) \neq 0$, is a restriction of an invariant and does not have the above described form. Therefore, $\alpha \cdot l$ does not belong to the subalgebra generated by supertraces of finite dimensional representations.

1.4. Proposition. Let $\mathfrak{g}=\bigoplus_{-1 \leq i \leq N} \mathfrak{g}_{i}$ be a consistent grading of a $\mathbb{Z}$-graded Lie superalgebra; moreover, let $\mathfrak{g}_{0}$ be reductive. Let $W=W\left(\mathfrak{g}_{0}\right)$ be the Weyl group of $\mathfrak{g}_{0}$ and $\alpha_{1}, \ldots, \alpha_{n}$ be the weights of the $\mathfrak{g}_{0}$-module $\mathfrak{g}_{-1}$.

Then any polynomial of the form $\alpha_{1} \ldots \alpha_{n} f$ on the Cartan subalgebra $\mathfrak{h}$ of $\mathfrak{g}_{0}$, where $f \in \mathbb{C}\left[\mathfrak{h}^{*}\right]^{W}$, is a restriction of an invariant.

Proof. Let $\lambda$ be a highest weight for $\mathfrak{g}_{0}, L^{\lambda}$ the corresponding irreducible $\mathfrak{g}_{0}$-module (with even heighest weight vector). Let $\mathfrak{g}_{i} L^{\lambda}=0$ for $i>0$; denote $\mathfrak{g}_{\geq 0}=\bigoplus_{i \geq 1} \mathfrak{g}_{i}$ and set $V^{\lambda}=\operatorname{ind}_{\mathfrak{g}_{\geq 0}}^{\mathfrak{g}}\left(L^{\lambda}\right)$. Then

$$
\operatorname{sch} V^{\lambda}=\prod_{1 \leq i \leq n}\left(1-e^{\alpha_{i}}\right) \operatorname{ch} L^{\lambda} .
$$

According to [B] there exists a linear combination $\sum c_{\lambda} \operatorname{ch} L^{\lambda}$ of functions $\operatorname{ch} L^{\lambda}$ such that the homogeneous component of the least degree of this combination is equal to $f$. But then the homogeneous component of the least degree of $\prod_{1 \leq i \leq n}\left(1-e^{\alpha_{i}}\right) \sum c_{\lambda} \operatorname{ch} L^{\lambda}$ is equal to $\alpha_{1} \ldots \alpha_{n} f$.

1.5. Proposition. Let $\mathfrak{g}=\mathfrak{g}_{\overline{0}} \oplus \mathfrak{g}_{\overline{1}}$ be a Lie superalgebra with reductive $\mathfrak{g}_{\overline{0}}$. Let $W=W\left(\mathfrak{g}_{\overline{0}}\right)$ be the Weyl group of $\mathfrak{g}_{\overline{0}}$ and $\alpha_{1}, \ldots, \alpha_{n}$ be the weights of the $\mathfrak{g}_{\overline{0}}$-module $\mathfrak{g}_{\overline{1}}$.

Then any polynomial of the form $\alpha_{1} \ldots \alpha_{n} f$ on the Cartan subalgebra $\mathfrak{h}$ of $\mathfrak{g}_{\overline{0}}$, where $f \in \mathbb{C}\left[\mathfrak{h}^{*}\right]^{W}$, is a restriction of an invariant.

Proof is similar to that of Proposition 1.4.

1.6. Proposition. Let $\mathfrak{g}=\mathfrak{s l}(2), L^{n}$ the finite dimensional irreducible $\mathfrak{g}$-module with highest weight $n \varphi$, where $\varphi$ is the fundamental weight.

Then for any $k \in \mathbb{N}$ and any $n_{1}, \ldots, n_{k+1} \in \mathbb{N}$ there exist $c_{1}, \ldots, c_{k+1} \in \mathbb{C}$ such that the homogeneous component of the least degree of the linear combination of characters $\sum c_{i} \operatorname{ch} L^{n_{i}}$ under the restriction on the Cartan subalgebra becomes equal to $\varphi^{2 k}$.

Proof. Since

$$
\operatorname{ch} L^{n_{i}}=\frac{\sinh \left(n_{i}+1\right) \varphi}{\sinh \varphi}
$$


it suffices to prove the statement for a linear combination $\sum c_{i} \sinh \left(n_{i}+1\right) \varphi$. By equating the coefficients of the powers $1,3, \ldots, 2 k-1$ to zero and equating the coefficient of the power $2 k+1$ to 1 we get the system

$$
\begin{aligned}
& c_{1}\left(n_{1}+1\right)+\cdots+c_{k+1}\left(n_{k+1}+1\right)=0 \\
& c_{1}\left(n_{1}+1\right)^{3}+\cdots+c_{k+1}\left(n_{k+1}+1\right)^{3}=0
\end{aligned}
$$

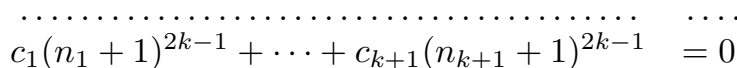

$$
\begin{aligned}
& c_{1}\left(n_{1}+1\right)^{2 k+1} \cdots+c_{k+1}\left(n_{k+1}+1\right)^{2 k+1}=(2 k+1) \text { ! }
\end{aligned}
$$

If the $n_{i}$ are pairwise distinct, the determinant of the system is nonzero.

Remark. Proposition 1.6 implies that

$$
\sum_{1 \leq i \leq k+1} c_{i} \sinh \left(n_{i} x\right) \varphi=x^{2 k+1}+\ldots
$$

By differentiating this identity with respect to $x$ we see that there exist $c_{1}^{\prime}, \ldots c_{k+1}^{\prime}$ such that

$$
\sum_{1 \leq i \leq k+1} c_{i}^{\prime} \cosh \left(n_{i} x\right) \varphi=x^{2 k}+\ldots
$$

1.7. The algebra of dual numbers and modules over associative superalgebras. Let $A$ be an associative superalgebra. Consider the algebra (with any parity ignored)

$$
A[\varepsilon]=\left\{a+b \varepsilon \mid a, b \in A, \varepsilon^{2}=1, \varepsilon a=(-1)^{p(a)} a \varepsilon\right\} .
$$

Observe that our algebra $A[\varepsilon]$ is almost the same as the superalgebra $Q(A)$ considered in [BL2], but (1) we ignore parity, $(2)$ in $Q(A)$ a different relation, $\varepsilon^{2}=-1$, should be satisfied.

1.7.1. Lemma. The category Mods of $A[\varepsilon]$-modules is isomorphic to the category SMods of graded A-modules, the morphisms of the latter being the purely even homomorphisms.

Proof. Let us construct the functors

$$
F: \text { Mods } \longrightarrow \text { SMods and } G: \text { SMods } \longrightarrow \text { Mods. }
$$

Let $V \in \operatorname{ObMods}$, then $\varepsilon \in \operatorname{End}(V)$ and since $\varepsilon^{2}=1$, it follows that $F(V)=V_{\overline{0}} \oplus V_{\overline{1}}$, where

$$
V_{\overline{0}}=\{v \in V \mid \varepsilon v=v\} \text { and } V_{\overline{1}}=\{v \in V \mid \varepsilon v=-v\} .
$$

Thus, $V$ may be considered as an object from SMods. It is an easy and routine to check, with the help of the commutation relations for $\varepsilon$, that if $f: V \longrightarrow W$ is an $A[\varepsilon]$-module homomorphism, then $f$ may be considered as an even homomorphism (morphism) of $A$-modules.

Every superspace $V=V_{\overline{0}} \oplus V_{\overline{1}} \in$ ObSMods has a fixed map (not a morphism), namely the parity map $J$ such that $J(v)=(-1)^{p(v)} v$; see [Be2]. Sending $\varepsilon$ to $J$ we get an object from Mods.

To verify that $F G$ and $G F$ are identity functors is trivial.

1.7.2. Lemma. Let $A$ be an associative superalgebra and $L(A)$ the corresponding Lie algebra. Set

$$
a * b=(-1)^{p(a)} a b-(-1)^{p(a) p(b)} b a .
$$


With respect to the action $*$ the space $A$ is an $L(A)$-module. Moreover, let $V$ be a graded $A$-module. The function $a \mapsto \operatorname{tr}_{V}(a)$ is an $L(A)$-invariant function on $A$.

Proof. Consider $A[\varepsilon]$ as a superalgebra with $p(\varepsilon)=\overline{0}$. (Observe that in [BL2] $p(\varepsilon)=$ 1.) Let us calculate $[a, \varepsilon b]$, where $a, b \in A$ and $[\cdot, \cdot]$ denotes the supercommutator. We have

$$
[a, \varepsilon b]=a \cdot \varepsilon b-(-1)^{p(a) p(b)} \varepsilon b a=\varepsilon\left((-1)^{p(a)} a b-(-1)^{p(a) p(b)} b a\right),
$$

which leads to the above formula for $a * b$.

Further on, $V$ is a graded $A[\varepsilon]$-module (since $J$ is an even map); hence by the characteristic property of the supertrace $\operatorname{str}[a, \varepsilon b]=0$; hence,

$$
\operatorname{tr}(a * b)=\operatorname{str}(\varepsilon a * b)=\operatorname{str}[a, \varepsilon b]=0
$$

(because if $a \in A$, then $\operatorname{str}(a)=\operatorname{tr}(\varepsilon a)$ and $\operatorname{tr}(a)=\operatorname{str}(\varepsilon a)$ ).

Remark. We are forced to introduce $A[\varepsilon]$ in its latter interpretation because we wish to consider not only the intertwining operators that become scalars on the irreducible modules but may become a scalar multiple of $J$. This train of thought leads us to the notion of anticenter considered elsewhere.

\section{INVARIANT POLYNOMIALS ON LIE SUPERALGEBRAS}

Let $\mathfrak{g}$ be one of the Lie superalgebras

$$
\mathfrak{g l}, \mathfrak{s l}, \mathfrak{p s l}, \mathfrak{o s p}, \mathfrak{p e}, \mathfrak{s p e}, \mathfrak{o s p}_{\alpha}(4 \mid 2) ; \quad \mathfrak{a g}_{2}, \mathfrak{a b}_{3} ; \quad \mathfrak{v e c t}, \mathfrak{s v e c t}, \widetilde{\mathfrak{s v e c t}}
$$

Let $\mathfrak{h}$ be the split Cartan subalgebra of $\mathfrak{g}$; let $R=R^{+} \cup R^{-}$be the set of nonzero roots of $\mathfrak{g}$ divided into subsets of positive and negative ones. Let $R_{0}$ and $R_{1}$, the subsets of even and odd roots; each set can be divided into the subsets $R_{0}^{ \pm}, R_{1}^{ \pm}$of positive and negative roots. We further set:

$$
\tilde{R}^{+}=\left\{\alpha \in R^{+} \mid-\alpha \in R^{-}\right\} \quad \tilde{R}_{1}^{+}=\left\{\alpha \in R_{1}^{+} \mid-\alpha \in R_{1}^{-} ; 2 \alpha \notin R_{1}^{+}\right\} .
$$

Let $\mathfrak{g}=\mathfrak{h} \oplus_{\alpha \in R} \mathfrak{g}^{\alpha}$ be the weight decomposition; for $\alpha \in \tilde{R}_{1}^{+}$set

$$
\nu(\alpha)=\min \left(\operatorname{dim} \mathfrak{g}^{\alpha}, \operatorname{dim} \mathfrak{g}^{-\alpha}\right) .
$$

For the Lie superalgebras considered $\nu(\alpha)=1$ or 2 . We set

$I^{\alpha}\left(\mathfrak{h}^{*}\right)=\left\{\begin{array}{l}\left\{f \in S\left(\mathfrak{h}^{*}\right) \mid D_{h} f \in(\alpha) \text { for } h \in\left[\mathfrak{g}_{\overline{1}}^{-\alpha}, \mathfrak{g}_{\overline{1}}^{\alpha}\right]\right\} \text { if } \nu(\alpha)=1, \\ \left\{f \in S\left(\mathfrak{h}^{*}\right) \mid D_{h} f \in(\alpha) \text { for } h \in\left[\mathfrak{g}_{\overline{1}}^{-\alpha}, \mathfrak{g}_{\overline{1}}^{\alpha}\right],\right. \\ \left.\text { and } D_{h_{1}} D_{h_{2}} f \in\left(\alpha^{2}\right) \text { for } h_{1}, h_{2} \text { that generate }\left[\mathfrak{g}_{\overline{1}}^{-\alpha}, \mathfrak{g}_{\overline{1}}^{\alpha}\right]\right\} \text { if } \nu(\alpha)=2 .\end{array}\right.$

Let $W$ be the Weyl group of $\mathfrak{g}_{\overline{0}}$ if $\mathfrak{g}_{\overline{0}}$ is reductive and the Weyl group of $\mathfrak{g}_{0}$ if $\mathfrak{g}$ is a vectorial Lie superalgebra (with nonreductive $\mathfrak{g}_{\overline{0}}$ ) in the standard $\mathbb{Z}$-grading.

Theorem. The restriction homomorphism $S\left(\mathfrak{g}^{*}\right) \longrightarrow S\left(\mathfrak{h}^{*}\right)$ induces an isomorphism of $S\left(\mathfrak{g}^{*}\right)^{\mathfrak{g}}$ with the algebra

$$
I\left(\mathfrak{h}^{*}\right)=\left\{f \in S\left(\mathfrak{h}^{*}\right)^{W} \mid f \in I^{\alpha}\left(\mathfrak{h}^{*}\right) \text { for any } \alpha \in \tilde{R}_{1}^{+}\right\} .
$$

Proof. Let us prove that the image $f$ of any invariant polynomial $F$ belongs to $I\left(\mathfrak{h}^{*}\right)$. First, observe that $f \in S\left(\mathfrak{h}^{*}\right)^{W}$.

Define $\mathfrak{g}_{\alpha}$ to be the Lie subsuperalgebra of $\mathfrak{g}$ generated by the root vectors of weights proportional to $\alpha$. Clearly, if $\mathfrak{g}_{\overline{0}}$ is reductive, $\mathfrak{g}_{\alpha}$ is isomorphic to one of the Lie (super)algebras from Proposition 1.2 if $\nu(\alpha)=1$ or one of the Lie (super)algebras from Proposition 1.3 if $\nu(\alpha)=2$ and $\mathfrak{g}=\mathfrak{s l}(2 \mid 2), \mathfrak{p s l}(2 \mid 2)$ or $\mathfrak{s p e}(4)$. 
Observe that the restriction of $F$ onto $\mathfrak{g}_{\alpha}$ belongs to $S\left(\mathfrak{g}_{\alpha}^{*}\right)^{\mathfrak{g}_{\alpha}}$. From Propositions 1.2 and 1.3 we deduce that $f \in I^{\alpha}\left(\mathfrak{h}^{*}\right)$.

If $\mathfrak{g}$ is of vectorial type, then $\nu(\alpha)=1$ and by selecting $u \in \mathfrak{g}^{-\alpha}, v \in \mathfrak{g}^{\alpha}$ we obtain a subalgebra $\mathfrak{b}=\mathfrak{h} \oplus \operatorname{Span}(u, v)$ satisfying the conditions of Proposition 1.2. Hence, in this case $f \in I^{\alpha}\left(\mathfrak{h}^{*}\right)$ as well.

Moreover, Proposition 1.1 shows that the restriction homomorphism is injective. Let us prove now that each element from $I\left(\mathfrak{h}^{*}\right)$ can be extended to an element from $S\left(\mathfrak{g}^{*}\right)^{\mathfrak{g}}$. The proof will be carried out separately for each of the above Lie superalgebras.

$\mathfrak{g}=\mathfrak{g l}(n \mid m)$. (cf. [B].) On the Cartan subalgebra, consider the formal power series $\overline{\mathbb{C}}\left[\left[\mathfrak{h}^{*}\right]\right]$. For $h \in \mathfrak{h}, \lambda \in \mathfrak{h}^{*}$ and $e^{\lambda} \in \mathbb{C}\left[\left[\mathfrak{h}^{*}\right]\right]$ set

$$
D_{h} e^{\lambda}=\lambda(h) e^{\lambda} \text {. }
$$

Define

$$
\begin{aligned}
& J(\mathfrak{h})^{*}=\left\{f \in \mathbb{C}\left[\left[\mathfrak{h}^{*}\right]\right]^{W} \mid f \text { is a linear combination of the } e^{\lambda},\right. \\
& \text { where } \lambda \text { is a weight of a representation of } \mathfrak{g}_{\overline{0}} \text { and }
\end{aligned}
$$$$
\left.D_{h} f \in(\alpha) \text { for any } \alpha \in R_{\overline{1}} \text { and } h \in\left[\mathfrak{g}_{\overline{1}}^{-\alpha}, \mathfrak{g}_{\overline{1}}^{\alpha}\right]\right\} \text {. }
$$

Let us prove that any element from $J\left(\mathfrak{h}^{*}\right)$ is a linear combination of supercharacters of finite dimensional representations. Indeed, since the homogeneous components of supercharacters are invariant polynomials, it follows that any linear combination of them belongs to $J(\mathfrak{h})^{*}$. Let $\left(\mathfrak{g}_{\overline{0}}\right)_{s}$ be the semisimple part of $\mathfrak{g}_{\overline{0}}$ and $\langle\cdot, \cdot\rangle$ the Killing form on $\left(\mathfrak{g}_{\overline{0}}\right)_{s}$.

For $\lambda \in \mathfrak{h}^{*}$ let $\lambda_{s}$ be its restriction onto $\mathfrak{h} \cap\left(\mathfrak{g}_{0}\right)_{s}$; we set

$$
|\lambda|^{2}=\left\langle\lambda_{s}, \lambda_{s}\right\rangle
$$

For $f=\sum c_{\nu} e^{\nu}$ set $r_{f}=\max _{\nu \neq 0}\left|\nu_{s}\right|$ and induct on $r_{f}$.

If $r_{f}=0$, then $\nu_{s}=0$ for any $\nu$. Let $\alpha \in R_{\overline{1}}$ and $h \in\left[\mathfrak{g}_{\overline{1}}^{-\alpha}, \mathfrak{g}_{\overline{1}}^{\alpha}\right]$. If $\nu(h)=0$, then $\nu_{s}=0$ implies that $\nu$ is proportional to the supertrace, the supercharacter of a one-dimensional representation. Therefore, we may assume that $\nu(h) \neq 0$ for any $\nu$.

Consider the restriction of $D_{h} f$ onto $\left[\mathfrak{g}_{\overline{1}}^{-\alpha}, \mathfrak{g}_{\overline{1}}^{\alpha}\right]$. Since $\alpha(h)=0$, it follows that $D_{h} f=\sum c_{\nu} \nu(h) e^{\nu}=0$. Since the exponents are linearly independent, $c_{\nu} \nu(h)=0$ for all $\nu$. And since $\nu(h) \neq 0$, it follows that each $c_{\nu}=0$; hence, $f=0$. Thus, if $\left|\nu_{s}\right|=0$, then $f=\sum c_{\nu} e^{\nu}$, where each $\nu$ is proportional to the supertrace. Let $r_{f}>0$ and $f=\sum c_{\nu} e^{\nu}$.

Consider the difference $f-\sum c_{\nu} \operatorname{sch} L^{\nu}$, where $L^{\nu}$ is the irreducible highest weight module with the highest weight $\nu$ and the sum runs over $\nu$ such that $\left|\nu_{s}\right|=r_{f}$. Since the difference is $W$-invariant, it contains summands $e^{\mu}$ with $\left|\mu_{s}\right|<r_{f}$ by a property of representations of semisimple Lie algebras (see $[\mathrm{B}]$ ). So the inductive hypothesis applies.

Now, let $P \in I\left(\mathfrak{h}^{*}\right)$; consider $P$ as a polynomial in weights of the standard module, i.e., $P=P\left(\varepsilon_{1}, \ldots, \varepsilon_{n} ; \delta_{1}, \ldots, \delta_{m}\right)$ and let

$$
f=P\left(e^{\varepsilon_{1}}-1, \ldots, e^{\varepsilon_{n}}-1 ; e^{\delta_{1}}-1, \ldots, e^{\delta_{m}}-1\right) .
$$


Let us verify that $f \in J\left(\mathfrak{h}^{*}\right)$. Clearly, $f$ is $W$-invariant. Let $\alpha=\varepsilon_{i}-\delta_{j}$ and $h \in\left[\mathfrak{g}_{\overline{1}}^{-\alpha}, \mathfrak{g}_{\overline{1}}^{\alpha}\right] ;$ then the condition $D_{h} f \in(\alpha)$ is equivalent to the fact that

$$
\begin{aligned}
\frac{\partial f}{\partial \varepsilon_{i}}+ & \frac{\partial f}{\partial \delta_{j}} \in\left(\varepsilon_{i}-\delta_{j}\right) \Longleftrightarrow \frac{\partial P}{\partial \varepsilon_{i}} e^{\varepsilon_{i}}+\frac{\partial P}{\partial \delta_{j}} e^{\delta_{j}} \in\left(e^{\varepsilon_{i}}-e^{\delta_{j}}\right) \\
& \Longleftrightarrow e^{\delta_{j}}\left(\frac{\partial P}{\partial \varepsilon_{i}} e^{\varepsilon_{i}-\delta_{j}}+\frac{\partial P}{\partial \delta_{j}}\right) \in\left(e^{\varepsilon_{i}-\delta_{j}}-1\right) e^{\delta_{j}} \\
& \Longleftrightarrow\left(\frac{\partial P}{\partial \varepsilon_{i}} e^{\varepsilon_{i}-\delta_{j}}+\frac{\partial P}{\partial \delta_{j}}\right) \in\left(e^{\varepsilon_{i}-\delta_{j}}-1\right) .
\end{aligned}
$$

Since $\frac{\partial P}{\partial \varepsilon_{i}}+\frac{\partial P}{\partial \delta_{j}} \in\left(\varepsilon_{i}-\delta_{j}\right)$, the last membership implies that $\frac{\partial f}{\partial \varepsilon_{i}}+\frac{\partial f}{\partial \delta_{j}} \in\left(\varepsilon_{i}-\delta_{j}\right)$. Since $f \in J\left(\mathfrak{h}^{*}\right)$, any homogeneous component of $f$ is a restriction of an invariant; but $P$ is an homogeneous component of $f$ of the least degree, hence, it is a restriction of an invariant.

$\underline{\mathfrak{g}}=\mathfrak{s l}(n \mid m), n \neq m$. Let $\mathfrak{h}$ the Cartan subalgebra of $\mathfrak{g l}(n \mid m)$; let $\tilde{\mathfrak{h}}=\mathfrak{h} \cap \mathfrak{g}$. Let $\overline{f \in I\left(\tilde{\mathfrak{h}}^{*}\right) ; \text { set } g(h)}=f\left(h-\frac{\operatorname{str} h}{n-m} 1_{n+m}\right)$. Then

$$
\left(D_{h_{\alpha}} g\right)(h)=\left(D_{h_{\alpha}} f\right)\left(h-\frac{\operatorname{str} h}{n-m} 1_{n+m}\right) \in \alpha\left(h-\frac{\operatorname{str} h}{n-m} 1_{n+m}\right)=\alpha(h) .
$$

Hence, $g \in I\left(\tilde{\mathfrak{h}}^{*}\right)$ and by the above is a restriction of an invariant. Hence, $f$ is also a restriction of an invariant.

$\mathfrak{g}=\mathfrak{s l}(n \mid n), n \neq 2$. Let us show that the restriction map $I\left(\mathfrak{h}^{*}\right) \longrightarrow I\left(\tilde{\mathfrak{h}}^{*}\right)$ is surjective. Let us identify $S\left(\mathfrak{h}^{*}\right)$ with $\mathbb{C}\left[\varepsilon_{1}, \ldots, \varepsilon_{n} ; \delta_{1}, \ldots, \delta_{m}\right]$, where $\varepsilon_{1}, \ldots, \varepsilon_{n} ; \delta_{1}, \ldots, \delta_{m}$ are the weights of the identity representation. It is not difficult to show (see $[\mathrm{P}]$ ) that

$$
I\left(\mathfrak{h}^{*}\right) \cong \mathbb{C}\left[s_{1}, s_{2}, \ldots\right], \text { where } s_{k}=\sum \varepsilon_{i}^{k}-\sum \delta_{j}^{k} \text { are supertraces of id. }
$$

Let $\tilde{\varepsilon}_{i}$ and $\tilde{\delta}_{j}$ be the images of $\varepsilon_{i}$ and $\delta_{j}$ in $\left.S\left(\tilde{\mathfrak{h}}^{*}\right)\right)$. Since the $\tilde{\varepsilon}_{i}$ and $\tilde{\delta}_{j}$ for $j<n$ are algebraically independent, we deduce, as above, that

$$
I\left(\tilde{\mathfrak{h}}^{*}\right) \subset \mathbb{C}\left[\sigma_{1}, \sigma_{2}, \ldots\right], \text { where } \sigma_{k}=\sum \tilde{\varepsilon}_{i}^{k}-\sum \tilde{\delta}_{j}^{k} .
$$

If $f \in I\left(\tilde{\mathfrak{h}}^{*}\right)$, then $f=F\left(\sigma_{1}, \sigma_{2}, \ldots\right)$. Consider $d=f-F\left(\sigma_{1}-\sigma_{1}, \sigma_{2}-\sigma_{1}^{2}, \ldots\right)$. Clearly, $d=0$ at $\sigma_{1}=0$; hence, $d$ is divisible by $\sigma_{1}$. Since $F\left(\sigma_{1}-\sigma_{1}, \sigma_{2}-\sigma_{1}^{2}, \ldots\right)$ is the image of an element from $I\left(\mathfrak{h}^{*}\right)$, we may assume that $f$ is divisible by $\sigma_{1}$, i.e., $f=\sigma_{1} g$.

For $\alpha=\varepsilon_{n}-\delta_{n}$ the condition $D_{h_{\alpha}} f \in(\alpha)$ is equivalent to the fact that the restriction of $f$ onto ker $\alpha$ is invariant with respect to translations by the vectors $h_{\alpha}$. Since $\sigma_{1}\left(h_{\alpha}\right) \neq 0$, any element from ker $\alpha$ can be expressed in the form $h+t h_{\alpha}$, where $h \in \operatorname{ker} \alpha \cap \operatorname{ker} \sigma_{1}$. Therefore,

$$
f\left(h+t h_{\alpha}\right)=\sigma_{1}\left(h+t h_{\alpha}\right) g\left(h+t h_{\alpha}\right)=t \sigma_{1}\left(h_{\alpha}\right) g\left(h+t h_{\alpha}\right)=f(h)=0 .
$$

Therefore, $g\left(h+t h_{\alpha}\right)=0$ and the restriction of $f$ onto ker $\alpha$ is equal to 0 . Hence, $f$ is divisible by $\alpha$; hence, from the $W$-symmetry and the fact that the linear functions $w \alpha$ are pairwise relatively prime for $n>2$ we deduce that

$$
f=\prod_{\alpha \in R_{1}^{+}} \alpha \cdot \tilde{\varphi}, \text { where } \tilde{\varphi}, \in \mathbb{C}\left[\tilde{\mathfrak{h}}^{*}\right]^{W}
$$


It is clear that $f$ is a restriction of an element of the form

$$
\prod_{\alpha \in R_{\overline{1}}^{+}} \alpha \cdot \varphi, \text { where } \varphi, \in \mathbb{C}\left[\mathfrak{h}^{*}\right]^{W} .
$$

Proposition 1.4 applied to $\mathfrak{g}=\mathfrak{g l}(n \mid n)=\mathfrak{g}_{-} \oplus \mathfrak{h} \oplus \mathfrak{g}_{+}$, where $\mathfrak{g}_{ \pm}$is the linear span of the positive (negative) root vectors, implies that $\prod_{\alpha \in R_{\overline{1}}^{+}} \alpha \cdot \varphi$ is the restriction of an invariant.

$\mathfrak{g}=\mathfrak{s l}(2 \mid 2)$. In this case the root spaces are two-dimensional. A direct calculation proves that $I\left(\mathfrak{h}^{*}\right)$ consists of the polynomials of the form

$$
c+\alpha_{1} \alpha_{2} g+\alpha_{1}^{2} \alpha_{2}^{2} \varphi
$$

where $c \in \mathbb{C}, g$ is a linear combination of the functions of the form $\frac{\tilde{\varepsilon}_{1}^{n}-\tilde{\varepsilon}_{2}^{n}}{\tilde{\varepsilon}_{1}-\tilde{\varepsilon}_{2}}$ for the even weights $\tilde{\varepsilon}_{1}$ and $\tilde{\varepsilon}_{2}$ of the identity $\mathfrak{g}$-module and $\varphi \in S\left(\mathfrak{h}^{*}\right)^{W}$.

Proposition 1.4 implies that $\alpha_{1}^{2} \alpha_{2}^{2} \varphi$ is the restriction of an invariant. To show that $\alpha_{1} \alpha_{2} g$ is the restriction of an invariant, consider the function $F(t)=\frac{\left(t-\tilde{\delta}_{1}\right)\left(t-\tilde{\delta}_{2}\right)}{\left(t-\tilde{\varepsilon}_{1}\right)\left(t-\tilde{\varepsilon}_{2}\right)}$, where $\tilde{\delta}_{1}$ and $\tilde{\delta}_{2}$ are odd weights of the identity $\mathfrak{g}$-module. The coefficients of the power series expansion in $t$ of $F(t)$ are expressed in terms of the supertraces of powers of the identity representation; hence, are restrictions of invariants.

Let $F(t)=\sum_{k \geq 0} t^{-k} \mu_{k}$. It is easy to check that $\mu_{k+2}=\alpha_{1} \alpha_{2} \frac{\tilde{\varepsilon}_{1}^{k}-\tilde{\varepsilon}_{2}^{k}}{\tilde{\varepsilon}_{1}-\tilde{\varepsilon}_{2}}$.

$\mathfrak{g}=\mathfrak{p s} \mathfrak{l}(n \mid n), n>1$. Let $\mathfrak{h} \subset \mathfrak{s l}(n \mid n)$ be the Cartan subalgebra, $\tilde{\mathfrak{h}}=\mathfrak{h} / \mathbb{C} \cdot z$, where $z$ is an element from the center of $\mathfrak{s l}(n \mid n)$. It is not difficult to verify that $I\left(\tilde{\mathfrak{h}}^{*}\right)$ can be embedded into $I\left(\mathfrak{h}^{*}\right)$ and the image coincides with the set of elements from $I\left(\mathfrak{b}^{*}\right)$ invariant under translations in the direction of $z$, i.e., $f$ such that $f(h+t z)=f(h)$.

Let us continue such a polynomial $f$ to an invariant $F$ from $S\left(\mathfrak{s l}(n \mid n)^{*}\right)^{\mathfrak{s l}(n \mid n)}$. Then $F$ also is invariant under translations in the direction of $z$; hence, determines an element from $S\left(\mathfrak{g}^{*}\right)^{\mathfrak{g}}$ whose restriction is equal to $f$. To establish this, it suffices to verify that the derivative in the direction of $z$ commutes with the restriction homomorphism onto $\tilde{\mathfrak{h}}$.

Observe that though there is a nondegenerate invariant supersymmetric even bilinear form on $\mathfrak{g}$, by Remark 1.3 this form is NOT related with any finite dimensional representation of $\mathfrak{g}$.

$\mathfrak{g}=\mathfrak{o s p}(2 \mid 2 n-2), n>1$. This Lie superalgebra possesses a compatible $\mathbb{Z}$-grading of depth 1; hence, there is a one-to-one correspondence between irreducible finite dimensional representations of $\mathfrak{g}$ and irreducible finite dimensional representations of $\mathfrak{g}_{\hat{0}}$. Therefore, the arguments applyed for $\mathfrak{g l}(m \mid n)$ are applicable here as well.

Namely, the same as for $\mathfrak{g l}(m \mid n)$, define the algebra $J\left(\mathfrak{h}^{*}\right)=\left\{f \in \mathbb{C}\left[\left[\mathfrak{h}^{*}\right]\right]^{W} \mid f\right.$ is a finite linear combination of the $e^{\lambda}$ with exponents equal to the weights of finite dimensional $\mathfrak{g}_{0}$-modules and $D_{h} f \in(\alpha)$ for any $h \in\left[\mathfrak{g}_{\overline{1}}^{-\alpha}, \mathfrak{g}_{\overline{1}}^{\alpha}\right]$ and $\left.\alpha \in R_{\overline{1}}^{+}=\bar{R}_{\overline{1}}^{+} \cdot\right\}$

In the same lines as for $\mathfrak{g l}(m \mid n)$, we prove that any element from $J\left(\mathfrak{h}^{*}\right)$ is a linear combination of supercharacters. 
Let us prove now that any element from $I\left(\mathfrak{h}^{*}\right)$ is a homogeneous component of an element from $J\left(\mathfrak{h}^{*}\right)$. Indeed, let $P \in I\left(\mathfrak{h}^{*}\right)$; then $P=P\left(\varepsilon_{1}, \delta_{1}, \ldots, \delta_{n-1}\right)$, where $\varepsilon_{1}, \delta_{1}, \ldots, \delta_{n-1}$ are the weights of the standard $\mathfrak{g}$-module. Set

$$
f=P\left(\frac{e^{\varepsilon_{1}}-e^{-\varepsilon_{1}}}{2}, \frac{e^{\delta_{1}}-e^{-\delta_{1}}}{2}, \ldots, \frac{e^{\delta_{n-1}}-e^{-\delta_{n-1}}}{2}\right) .
$$

Let us check that $f \in J\left(\mathfrak{h}^{*}\right)$. Clearly, $f^{W}=f$. For $\alpha=\varepsilon_{1}-\delta_{1}$ the condition $D_{h} f \in(\alpha)$ is equivalent (because the restriction of the invariant form onto $\mathfrak{h}$ is proportional to $\left.\varepsilon_{1}^{2}-\sum \delta_{j}^{2}\right)$ to the fact that $\frac{\partial f}{\partial \varepsilon_{1}}+\frac{\partial f}{\partial \delta_{1}} \in\left(\varepsilon_{1}-\delta_{1}\right)$.

Since $P \in I\left(\mathfrak{h}^{*}\right)$, it follows that $\frac{\partial P}{\partial \varepsilon_{1}}+\frac{\partial P}{\partial \delta_{1}}=\left(\varepsilon_{1}-\delta_{1}\right) Q$. Hence,

$$
\begin{aligned}
\frac{\partial f}{\partial \varepsilon_{1}} & +\frac{\partial f}{\partial \delta_{1}}=\frac{\partial P}{\partial \varepsilon_{1}} \cosh \left(\varepsilon_{1}\right)+\frac{\partial P}{\partial \delta_{1}} \cosh \left(\delta_{1}\right) \\
& =\frac{\partial P}{\partial \varepsilon_{1}} \cosh \left(\varepsilon_{1}\right)+\left(\operatorname{sh}\left(\varepsilon_{1}\right)-\sinh \left(\delta_{1}\right)\right) Q \cosh \left(\delta_{1}\right)-\frac{\partial P}{\partial \varepsilon_{1}} \cosh \left(\delta_{1}\right) \\
& =\frac{\partial P}{\partial \varepsilon_{1}}\left(\cosh \left(\varepsilon_{1}\right)-\cosh \left(\delta_{1}\right)\right)+\left(\sinh \left(\varepsilon_{1}\right)-\sinh \left(\delta_{1}\right)\right) Q \cosh \left(\delta_{1}\right) \in(\alpha)
\end{aligned}
$$

Therefore, $f \in J\left(\mathfrak{h}^{*}\right)$ and its homogeneous component of the least degree - equal to $P$ - is the restriction of an invariant.

$\mathfrak{g}=\mathfrak{o s p}(2 m+1 \mid 2 n)$. For a basis of $\mathfrak{h}^{*}$ we take the weights $\varepsilon_{1}, \ldots, \varepsilon_{m} ; \delta_{1}, \ldots, \delta_{n}$ of the identity representation (of the two weights $\pm \varepsilon_{i}$ and $\pm \delta_{j}$ we select one). Observe that the odd roots $\pm \delta_{j}$ of $\mathfrak{g}$ are collinear to the even ones.

The Weyl group separately permutes the $\varepsilon$ 's and the $\delta$ 's and changes their signs. The restriction of the invariant form onto $\mathfrak{h}$ is proportional to $\sum \varepsilon_{i}^{2}-\sum \delta_{j}^{2}$; hence, for $\alpha=\varepsilon_{i}-\delta_{j}$ (observe that $\alpha \in \tilde{R}_{\overline{1}}$ ) the condition $D_{h} f \in(\alpha)$ is equivalent to the fact that $\frac{\partial f}{\partial \varepsilon_{i}}+\frac{\partial f}{\partial \delta_{j}} \in\left(\varepsilon_{i}-\delta_{j}\right)$, which, in turn, means that $f$ does not depend on $t$ after substitution $\varepsilon_{i}=\delta_{j}=t$. Clearly,

$$
f=f\left(\varepsilon_{1}^{2}, \ldots, \varepsilon_{m}^{2}, \delta_{1}^{2}, \ldots, \delta_{n}^{2}\right)
$$

and, therefore, is a supersymmetric polynomial in the sense of $[\mathrm{P}]$ and as such can be expressed via the coefficients of the rational function

$$
F(t)=\frac{\prod\left(t^{2}-\delta_{j}^{2}\right)}{\prod\left(t^{2}-\varepsilon_{i}^{2}\right)} .
$$

These coefficients are expressed via the sums $\sum_{i} \varepsilon_{i}^{k}-\sum_{j} \delta_{j}^{k}$ - the powers of the supertrace of the identity representation.

$\mathfrak{g}=\mathfrak{o s p}(2 m \mid 2 n)$. For a basis of $\mathfrak{h}^{*}$ we take the same basis $\varepsilon_{1}, \ldots, \varepsilon_{m} ; \delta_{1}, \ldots, \delta_{n}$ as in the preceding case. The restriction of any $W$-invariant polynomial is of the form

$$
f=P\left(\varepsilon_{1}^{2}, \ldots, \varepsilon_{m}^{2}, \delta_{1}^{2}, \ldots, \delta_{n}^{2}\right)+\varepsilon_{1} \cdots \varepsilon_{m} \cdot Q\left(\varepsilon_{1}^{2}, \ldots, \varepsilon_{m}^{2}, \delta_{1}^{2}, \ldots, \delta_{n}^{2}\right) .
$$

Moreover, the restriction of any $W$-invariant polynomial does not depend on $t$ after substitution $\varepsilon_{i}=\delta_{j}=t$, whereas after such a substitution $P$ is of an even degree with respect to $t$ and the second summand is of an odd degree. This means that both summands do not depend on $t$; hence, $P \in I\left(\mathfrak{h}^{*}\right)$ and $\varepsilon_{1} \ldots \varepsilon_{m} Q \in I\left(\mathfrak{h}^{*}\right)$ and

$$
Q=\prod\left(\varepsilon_{i}^{2}-\varepsilon_{i}^{2}\right) Q_{1} \text { for some polynomial } Q_{1} \text {. }
$$


The same arguments as in the preceding case show that $P$ is the restriction of an invariant. Let us prove that $\varepsilon_{1} \ldots \varepsilon_{m} Q$ is also the restriction of an invariant. To this end, select in $\mathfrak{h}$ the right dual basis $e_{1}, \ldots, e_{m} ; f_{1}, \ldots, f_{n}$ to $\varepsilon_{1}, \ldots, \varepsilon_{m}$; $\delta_{1}, \ldots, \delta_{n}$. For the system of simple roots take

$$
\delta_{1}-\delta_{2}, \ldots, \delta_{n-1}-\delta_{n} ; \delta_{n}-\varepsilon_{1}, \varepsilon_{1}-\varepsilon_{2}, \ldots, \varepsilon_{m-1}-\varepsilon_{m}, \varepsilon_{m-1}+\varepsilon_{m} .
$$

Let $\Lambda \in \mathfrak{h}^{*}$ be such that $\Lambda\left(e_{i}\right)=\lambda_{i}$ and $\Lambda\left(f_{j}\right)=\mu_{j}$. Then due to [K1] for the representation with highest weight $\Lambda$ to be a finite dimensional one, the coordinates of the highsest weight should satisfy the following conditions:

$$
\begin{aligned}
& \mu_{j} \in \mathbb{Z} \text { and } \mu_{1} \geq \mu_{2} \geq \cdots \geq \mu_{n} \geq m \\
& \lambda_{1}-\lambda_{2} \in \mathbb{Z}_{+}, \ldots, \lambda_{m-1}-\lambda_{m} \in \mathbb{Z}_{+}, \lambda_{m-1}+\lambda_{m} \in \mathbb{Z}_{+} .
\end{aligned}
$$

If $\Lambda$ satisfies $\lambda_{m} \neq 0$, then $\Lambda$ is a typical weight. Indeed, by Kac, [K1], the typicality condition is

$$
(\Lambda+\rho)\left(h_{\alpha}\right) \neq 0 \text { for any } \alpha \in R_{\overline{1}}^{+}
$$

or, equivalently,

$$
(\Lambda+\rho)\left(e_{i}\right) \neq 0 \text { for any } i \text { and }(\Lambda+\rho)\left(f_{j}\right) \neq 0 \text { for any } j .
$$

As is not difficult to verify,

$$
\rho_{0}=(m-1) \varepsilon_{1}+\cdots+\varepsilon_{m-1}+n \delta_{1}+\cdots+2 \delta_{n} ; \quad \rho_{1}=m \sum \delta_{i} ;
$$

hence,

$$
(\Lambda+\rho)\left(e_{i}\right)=\lambda_{i}+m-i \neq 0 \text { and }(\Lambda+\rho)\left(f_{j}\right)=\mu_{j}-m+m-j+1 \neq 0
$$

as was required for typicality.

Let $\Lambda$ be a typical weight. Then by [K2] the supercharacter of the irreducible module $L^{\Lambda}$ with the highest weight $\Lambda$ is

$$
\begin{aligned}
& \operatorname{sch} L^{\Lambda}=\frac{\prod_{\alpha \in R_{\overline{1}}^{+}}\left(e^{\alpha / 2}-e^{-\alpha / 2}\right)}{\prod_{\alpha \in R_{\overline{0}}^{+}}\left(e^{\alpha / 2}-e^{-\alpha / 2}\right)} \sum \varepsilon(w) e^{w(\Lambda+\rho)} \\
& =\prod_{\alpha \in R_{\overline{1}}^{+}}\left(e^{\alpha / 2}-e^{-\alpha / 2}\right) \prod_{\alpha \in R_{\overline{0}}^{+}}\left(e^{\alpha / 2}-e^{-\alpha / 2}\right)^{-1} \sum \varepsilon(w) e^{w\left(\Lambda+\rho_{0}-\rho_{1}\right)} \\
& =\prod_{\alpha \in R_{\overline{1}}^{+}}\left(e^{\alpha / 2}-e^{-\alpha / 2}\right) \operatorname{ch} L_{0}^{\Lambda-\rho_{1}}
\end{aligned}
$$

where $L_{0}^{\Lambda-\rho_{1}}$ is the irreducible $\mathfrak{g}_{\overline{0}}$-module with the highest weight $\Lambda-\rho_{1}$.

Consider $\Lambda$ such that $\lambda_{m} \neq 0$ and $\mu_{n} \geq m$, i.e., consider the highest weights of the typical irreducible finite dimensional modules. Then $\Lambda-\rho_{1}$ runs the highsest weights of $\mathfrak{g}_{\overline{0}}$ for which $\lambda_{m} \neq 0$. Let us demand that $\lambda_{m}$ are half-integer; then $\lambda_{m} \neq 0$ holds automatically. Let $T \in S\left(\mathfrak{h}_{\overline{0}}^{*}\right)^{W}$ be an invariant polynomial of the form

$$
T=\varepsilon_{1} \cdots \varepsilon_{m} \cdot Q_{1}\left(\varepsilon_{1}^{2}, \ldots, \varepsilon_{m}^{2}, \delta_{1}^{2}, \ldots, \delta_{n}^{2}\right) \text { for } Q_{1} \text { defined in }(*)
$$


Consider

$$
\begin{gathered}
\tilde{T}=T\left(e^{\varepsilon_{1} / 2}-e^{-\varepsilon_{1} / 2}, \ldots, e^{\varepsilon_{m} / 2}-e^{-\varepsilon_{m} / 2} ; e^{\delta_{1} / 2}-e^{-\delta_{1} / 2}, \ldots, e^{\delta_{n} / 2}-e^{-\delta_{n} / 2}\right) \\
=\prod_{i=1}^{m}\left(e^{\varepsilon_{i} / 2}-e^{-\varepsilon_{i} / 2}\right) \cdot S\left(2 \sinh \left(\varepsilon_{1} / 2\right), \ldots, 2 \sinh \left(\varepsilon_{m} / 2\right) ;\right. \\
\left.2 \sinh \left(\delta_{1} / 2\right), \ldots, 2 \sinh \left(\delta_{n} / 2\right)\right) .
\end{gathered}
$$

All the weights of the first factor are half-integer and linearly independent, hence, it is a linear combination of the $e^{\chi}$, where the coordinates $\chi_{i}$ are half-integers and nonzero (actually, this $\chi$ is the character of a spinor-like representation).

At the same time all the weights of the second factor are integer; so $T$ is a linear combination of the $e^{\chi}$ with $\chi_{m} \neq 0$ and half-integer. This expression is a linear combination of characters of finite dimensional representations

$$
\tilde{T}=\sum C_{\chi} \operatorname{ch} L_{0}^{\chi}
$$

of $\mathfrak{g}_{\overline{0}}$. Since all the weights $\mu$ that contribute to $S$ are half-integer with $\mu_{m} \neq 0$, then all the coordinates of $\chi$ are also half-integer and $\chi_{m} \neq 0$.

By multiplying both sides of the last equation by $L=\prod_{\alpha \in R_{\overline{1}}^{+}}\left(e^{\alpha / 2}-e^{-\alpha / 2}\right)$ we obtain

$$
L \cdot \tilde{T}=\sum C_{\chi} L \cdot \operatorname{ch} L_{0}^{\chi}=C_{\chi} \operatorname{sch} L^{\chi+\rho_{1}} .
$$

Therefore, the lowest component of $L \tilde{S}$ is the restriction of an invariant. But this lowest component is $\varepsilon_{1} \cdots \varepsilon_{m} \cdot Q\left(\varepsilon_{1}^{2}, \ldots, \varepsilon_{m}^{2}, \delta_{1}^{2}, \ldots, \delta_{n}^{2}\right)$.

$\mathfrak{g}=\mathfrak{o s p}_{\alpha}(4 \mid 2)$. In this case $\mathfrak{g}_{\overline{0}}=\mathfrak{s l}_{1}(2) \oplus \mathfrak{s l}_{2}(2) \oplus \mathfrak{s l}_{3}(2)$ (the sum of three copies of $\mathfrak{s l}(2)$, numbered to distinguish them) and $\mathfrak{g}_{\overline{1}}=V_{1} \otimes V_{2} \otimes V_{3}$, where $V_{i}$ is the identity representation of the $i$-th copy of $\mathfrak{s l}(2)$. Let $\pm \varepsilon_{1}, \pm \varepsilon_{2}, \pm \varepsilon_{3}$ be the weights of each of the components of $\mathfrak{g}_{\overline{0}}$ in its identity module. Then the root system of $\mathfrak{g}$ is as follows:

$$
R_{\overline{0}}=\left\{ \pm 2 \varepsilon_{1}, \pm 2 \varepsilon_{2}, \pm 2 \varepsilon_{3}\right\}, \quad R_{\overline{1}}=\left\{ \pm \varepsilon_{1} \pm \varepsilon_{2} \pm \varepsilon_{3}\right\} .
$$

The restriction of the nondegenerate invariant supersymmetric even bilinear form onto the Cartan subalgebra is of the form

$$
\frac{1}{\lambda_{1}} \varepsilon_{1}^{2}+\frac{1}{\lambda_{2}} \varepsilon_{2}^{2}+\frac{1}{\lambda_{3}} \varepsilon_{3}^{2}, \text { where } \lambda_{1}=-(1+\alpha), \lambda_{2}=1, \lambda_{3}=\alpha .
$$

From the condition $\left(h_{\alpha}, h\right)=\alpha(h)$ for $h \in \mathfrak{h}$ we deduce that if $\alpha=\theta_{1} \varepsilon_{1}+\theta_{2} \varepsilon_{2}+\theta_{3} \varepsilon_{3}$, where $\theta_{i}= \pm 1$, then

$$
h_{\alpha}=\theta_{1} \lambda_{1} H_{1}+\theta_{2} \lambda_{2} H_{2}+\theta_{3} \lambda_{3} H_{3}
$$

for the dual basis of $\mathfrak{h}$, i.e., $\varepsilon_{i}\left(H_{j}\right)=\delta_{i j}$.

A direct calculation shows that

$$
D_{h_{\alpha}} f=\theta_{1} \lambda_{1} \frac{\partial f}{\partial \varepsilon_{1}}+\theta_{2} \lambda_{2} \frac{\partial f}{\partial \varepsilon_{2}}+\theta_{3} \lambda_{3} \frac{\partial f}{\partial \varepsilon_{3}} .
$$

The Weyl group is isomorphic to $(\mathbb{Z} / 2)^{3}$; it acts by changing the signs of the $\varepsilon_{i}$. 
Let us describe now the algebra $I\left(\mathfrak{h}^{*}\right)$. An easy calculation shows that $I\left(\mathfrak{h}^{*}\right)$ consists of the elements of the form

$$
f=\psi\left(\frac{1}{\lambda_{1}} \varepsilon_{1}^{2}+\frac{1}{\lambda_{2}} \varepsilon_{2}^{2}+\frac{1}{\lambda_{3}} \varepsilon_{3}^{2}\right)+\prod_{\alpha \in R_{1}^{+}} \alpha \cdot \varphi,
$$

where $\varphi \in S\left(\mathfrak{h}^{*}\right)^{W}$. Clearly, the first summand is the restriction of an invariant. Let us prove that the second summand is also the restriction of an invariant.

For a system of simple roots take $\left\{\varepsilon_{1}+\varepsilon_{2}+\varepsilon_{3},-2 \varepsilon_{2},-2 \varepsilon_{3}\right\}$. Applying Proposition 1.5 to $\mathfrak{g}$ we see that any element of the form $\prod_{\alpha \in R_{1}^{+}} \alpha^{2} \cdot \varphi$, where $\varphi \in S(\mathfrak{h})^{W}$ is the restriction of an invariant. Since $\mathfrak{g}$ possesses a nondegenerate invariant supersymmetric even bilinear form and the Harish-Chandra homomorphism applies, any element from $S(\mathfrak{h})^{W}$ can be expressed in the form

$\frac{f}{Q^{2}}$, where $f$ is the image of an element from the center of $U(\mathfrak{g})$ and $Q=\prod_{\alpha \in R_{1}^{+}} h_{\alpha}$.

This implies that the irreducible finite dimensional $\mathfrak{g}$-module with highest weight $\Lambda$ is typical if

$$
\prod_{\alpha \in R_{1}^{+}} \Lambda\left(h_{\alpha}\right) \neq 0
$$

Remark. In [K2] this statement is conjectured, while the conditions sufficient for typicality of the modules over $\mathfrak{o s p}_{\alpha}(4 \mid 2), \mathfrak{a g}_{2}$ and $\mathfrak{a b}_{3}$ (these Lie superalgebras are differently baptized there) are faulty.

As for $\mathfrak{o s p}(2 n \mid 2 m)$, the formula for the supercharacter of the typical module can be expressed in the form

$$
\operatorname{sch} L^{\Lambda}=\prod_{\alpha \in R_{1}^{+}}\left(e^{\alpha / 2}-e^{-\alpha / 2}\right) \operatorname{ch} L_{0}^{\Lambda-\rho_{1}} .
$$

In the chosen system of simple roots

$$
\rho_{0}=\varepsilon_{1}-\varepsilon_{2}-\varepsilon_{3} ; \rho_{1}=2 \varepsilon_{1} .
$$

If $\Lambda=\chi_{1} \varepsilon_{1}+\chi_{2} \varepsilon_{2}+\chi_{3} \varepsilon_{3}$ is the highest weight, then $L^{\Lambda}$ is of finite dimension whenever

$$
\chi_{1} \geq 2, \chi_{1} \in \mathbb{Z}_{+} ; \quad \chi_{2}, \chi_{3} \in \mathbb{Z}_{-} ;
$$

the module $L^{\Lambda}$ is typical if additionally

$$
\prod_{\alpha \in R_{\overline{1}}^{+}} \Lambda\left(h_{\alpha}\right) \neq 0
$$

Fix $\chi_{2}$ and $\chi_{3}$. The equation for $\chi_{1}$ obtained has finitely many solutions. Hence, by selecting $\chi_{1}$ sufficiently large we deduce that for arbitrary $\chi_{2}$ and $\chi_{3}$ (such that $\chi_{2}, \chi_{3} \in \mathbb{Z}_{-}$) the module $L^{\Lambda}$ with $\Lambda=\chi_{1} \varepsilon_{1}+\chi_{2} \varepsilon_{2}+\chi_{3} \varepsilon_{3}$ is finite dimensional and typical.

Now, represent $\mathfrak{g}_{\overline{0}}$ in the form $\mathfrak{g}_{\overline{0}}=\mathfrak{s l}(2) \oplus \tilde{\mathfrak{g}}$ and set $\mathfrak{h}=\operatorname{Span}\left(H_{1}\right) \oplus \tilde{\mathfrak{h}}$, respectively. According to Bernstein [B], any element $P$ from $S\left(\tilde{\mathfrak{h}}^{*}\right)^{\mathbb{Z} / 2 \times \mathbb{Z} / 2}$ is the component of the least degree of the linear combination $T$ of characters of finite 
dimensional representations of $\tilde{\mathfrak{g}}$. By Proposition 1.6, $\varepsilon_{1}^{k}$ is a linear combination $\tilde{T}$ of characters of finite dimensional representations of $\mathfrak{s l}(2)$ whose highest weight is sufficiently big. Then the product $\varepsilon_{1}^{k} \cdot P$ is a linear combination of characters of finite dimensional representations of $\mathfrak{g}$ that enter $\tilde{T} \cdot T$ and each of which satisfies requirements for finite dimension and typicality.

Therefore, $\prod_{\alpha \in R_{\overline{1}}^{+}}\left(e^{\alpha / 2}-e^{-\alpha / 2}\right) \varepsilon_{1}^{k} \cdot P$ is a linear combination of supercharacters on $\mathfrak{g}$ and its lowest component is equal to $\prod_{\alpha \in R_{1}^{+}} \alpha \cdot \varepsilon_{1}^{k} \cdot P$.

$\mathfrak{g}=\mathfrak{a g}_{2}$. In this case $\mathfrak{g}_{\overline{0}}=\mathfrak{g}_{2} \oplus \mathfrak{s l}(2)$ and $\mathfrak{g}_{\overline{1}}=R\left(\pi_{1}\right) \otimes V$, where $R\left(\pi_{1}\right)$ is the first fundamental representation of $\mathfrak{g}_{2}$ (see [OV] or [Bu1]) and $V$ is the identity representation of $\mathfrak{s l}(2)$. We select a basis $H_{1}, H_{2}, H_{3}$, in the Cartan subalgebra of $\mathfrak{g}_{2}$ so that $H_{1}+H_{2}+H_{3}=0$; let the $\lambda_{i}$ be the linear forms such that $\lambda_{i}\left(H_{j}\right)=-1$ if $i \neq j$ and $\lambda_{i}\left(H_{i}\right)=2$.

Let $\pm \delta$ be the weights of the identity $\mathfrak{s l}(2)$-module; select the basis element $H$ of the Cartan subalgebra of $\mathfrak{s l}(2)$ so that $\delta(H)=1$. Then the root system of $\mathfrak{g}$ is as follows

$$
R_{\overline{0}}=\left\{\lambda_{i}-\lambda_{j} ; \pm \lambda_{i} ; \pm 2 \delta\right\}, \quad R_{\overline{1}}=\left\{ \pm \lambda_{i} \pm \delta ; \pm \delta\right\}
$$

For the system of simple roots select

$$
\lambda_{1}+\delta, \lambda_{2} ; \lambda_{3}-\lambda_{2}
$$

Then

$$
\begin{aligned}
& R_{\overline{0}}^{+}=\left\{\lambda_{2}, \lambda_{3},-\lambda_{1} ; \lambda_{2}-\lambda_{1}, \lambda_{3}-\lambda_{2}, \lambda_{3}-\lambda_{1}, 2 \delta\right\}, \\
& R_{\overline{1}}^{+}=\left\{\lambda_{1}+\delta, \lambda_{2}+\delta, \lambda_{3}+\delta,-\lambda_{1}+\delta,-\lambda_{2}+\delta,-\lambda_{3}+\delta, \delta\right\} .
\end{aligned}
$$

Observe that $\delta \in R_{\overline{1}}^{+}$while $2 \delta \in R_{\overline{0}}^{+}$; hence, $\tilde{R}_{\overline{1}}=R_{\overline{1}}^{+} \backslash\{\delta\}$. The Weyl group is isomorphic to $\left(S_{3} \circ \mathbb{Z} / 2\right) \times \mathbb{Z} / 2$, where $S_{3}$ permutes the $\lambda_{i}$, the first $\mathbb{Z} / 2$ simultaneusly changes the signs of all the $\lambda_{i}$, the second $\mathbb{Z} / 2$ changes the sign of $\delta$. It is not difficult to verify that

$$
S\left(\mathfrak{h}^{*}\right)^{W}=\mathbb{C}\left[\lambda_{1}^{2}+\lambda_{2}^{2}+\lambda_{3}^{2}, \quad\left(\lambda_{1} \lambda_{2} \lambda_{3}\right)^{2}, \quad \delta^{2}\right]
$$

The restriction of the nondegenerate invariant supersymmetric even bilinear form $\mathfrak{h}$ is proportional to

$$
3 \delta^{2}-2\left(\lambda_{1}^{2}+\lambda_{2}^{2}+\lambda_{3}^{2}\right) .
$$

From the condition $\left(h_{\alpha}, h\right)=\alpha(h)$ for $h \in \mathfrak{h}$ setting $\alpha=\lambda_{3}+\delta$ and selecting $H_{1}, H_{2}, H$ for a basis of $\mathfrak{h}$ we deduce that

$$
h_{\alpha}=-H_{1}-H_{2}-2 H \text {. }
$$

Therefore, the condition $D_{h_{\alpha}} f \in(\alpha)$ is equivalent to the following one:

$$
\frac{\partial f}{\partial \lambda_{1}}+\frac{\partial f}{\partial \lambda_{2}}+2 \frac{\partial f}{\partial \delta}+\in\left(\delta-\lambda_{1}-\lambda_{2}\right) .
$$

The other conditions of a similar type follow from $W$-invariance. Direct calculations demonstrate that $I\left(\mathfrak{h}^{*}\right)$ consists of the elements of the form

$$
f=\psi\left(3 \delta^{2}-2\left(\lambda_{1}^{2}+\lambda_{2}^{2}+\lambda_{3}^{2}\right)\right)+\prod_{\alpha \in \tilde{R}_{\overline{1}}^{+}} \alpha \cdot \varphi,
$$

where $\varphi \in S\left(\mathfrak{h}^{*}\right)^{W}$. 
The same arguments as in the study of $\mathfrak{o s p}_{\alpha}(4 \mid 2)$ prove with the help of Proposition 1.5 that if $\prod_{\alpha \in R_{\overline{1}}^{+}} \Lambda\left(h_{\alpha}\right) \neq 0$, then the module $L^{\Lambda}$ is a typical one. In this case the formula for the supercharacter can be expressed in the form ([K2])

$$
\operatorname{sch} L^{\Lambda}=\prod_{\alpha \in R_{\overline{1}}^{+}}\left(e^{\alpha / 2}-e^{-\alpha / 2}\right) \prod_{\alpha \in R_{0}^{+}}\left(e^{\alpha / 2}-e^{-\alpha / 2}\right)^{-1} \sum_{w \in W} \varepsilon^{\prime}(w) e^{w(\Lambda+\rho)},
$$

where $\varepsilon^{\prime}(w)=(-1)^{N(w)}$ and $N(w)$ is the parity of the number of reflections in all even roots except $2 \delta$.

Let $W_{1}=S_{2} \circ \mathbb{Z} / 2$ be the Weyl group of $\mathfrak{g}_{2}$; let $\rho_{0}{ }^{\prime}$ be the halfsum of the positive roots for $\mathfrak{g}_{2}$ and $\rho_{0}{ }^{\prime \prime}=\delta$ the halfsum of the positive roots for $\mathfrak{s l}(2)$; let $\rho_{1}=\frac{7}{2} \delta$ be the halfsum of the positive odd roots.

If $w$ is the reflection in $2 \delta$, then $\varepsilon^{\prime}(w)=1$. Therefore the formula for the supercharacter can be represented in the following form:

$$
\begin{aligned}
& \operatorname{sch} L^{\Lambda}=\prod_{\alpha \in \tilde{R}_{\overline{1}}^{+}}\left(e^{\alpha / 2}-e^{-\alpha / 2}\right) \prod_{\alpha \in \tilde{R}_{\overline{0}}^{+}}\left(e^{\alpha / 2}-e^{-\alpha / 2}\right)^{-1}\left(e^{\delta / 2}-e^{-\delta / 2}\right)\left(e^{\delta}-e^{-\delta}\right)^{-1} \\
& \times\left(\sum_{w \in W_{1}} \varepsilon(w) e^{w\left(\Lambda^{\prime}+\rho^{\prime}{ }_{0}\right)}\right) \cdot\left(e^{\Lambda^{\prime \prime}+\rho^{\prime \prime}{ }_{0}-\rho_{1}}+e^{-\left(\Lambda^{\prime \prime}+\rho^{\prime \prime}{ }_{0}-\rho_{1}\right)}\right) \\
& =\prod_{\alpha \in \tilde{R}_{\overline{1}}^{+}}\left(e^{\alpha / 2}-e^{-\alpha / 2}\right)\left(e^{\delta / 2}+e^{-\delta / 2}\right)^{-1} \operatorname{ch} L_{0}^{\Lambda^{\prime}}\left(e^{\Lambda^{\prime \prime}+\rho^{\prime \prime}{ }_{0}-\rho_{1}}+e^{-\left(\Lambda^{\prime \prime}+\rho^{\prime \prime}{ }_{0}-\rho_{1}\right)}\right),
\end{aligned}
$$

where $\Lambda^{\prime}$ is the restriction of $\Lambda$ onto the Cartan subalgebra of $\mathfrak{g}_{2}$ and $\Lambda^{\prime \prime}$ is the same for $\mathfrak{s l}(2)$ when

$$
\Lambda^{\prime \prime}(H) \geq 7 .
$$

Under this condition $\operatorname{dim} L^{\Lambda}<\infty$. To see this, it suffices to take the $\mathfrak{g}_{\overline{0}}$-module $\operatorname{ch} L_{0}^{\Lambda}$ with the same highest weight and the highest weight vector $v$; then in the induced module $\operatorname{Ind}_{\mathfrak{g}_{\overline{0}}^{\mathfrak{g}}}^{\mathfrak{g}} L_{0}^{\Lambda}$, consider the submodule generated by $\prod_{\alpha \in R_{1}^{+}} h_{\alpha} \cdot v$. (This submodule is currently called sometimes in the literature "Kac' module".)

Let $P \in I\left(\mathfrak{h}^{*}\right)$. Then, as we have shown,

$$
P=P_{1}\left(3 \delta^{2}-2\left(\lambda_{1}^{2}+\lambda_{2}^{2}+\lambda_{3}^{2}\right)\right)+\prod_{\alpha \in R_{\overline{1}}^{+}} \alpha \cdot P_{2},
$$

where $P_{2} \in S\left(\mathfrak{h}^{*}\right)^{W}$. As we have already mentioned, $3 \delta^{2}-2\left(\lambda_{1}^{2}+\lambda_{2}^{2}+\lambda_{3}^{2}\right)$ is the restriction of $\operatorname{str}$ ad $X$. Let us show that $\prod_{\alpha \in R_{1}^{+}} \alpha \cdot P_{2}$ is also the restriction of an invariant. Since $P_{2} \in S\left(\mathfrak{h}^{*}\right)^{W}$, we may assume that $P_{2}=Q_{2} \cdot \delta^{2 k}$, where $Q_{2} \in S\left(\left(\mathfrak{g}_{2} \cap \mathfrak{h}\right)^{*}\right)^{W_{1}}$.

According to Bernstein ([B]) $Q_{2}$ is the lowest component of a linear combination $T$ of characters of irreducible $\mathfrak{g}_{2}$-modules.

By Remark 1.6, $\delta^{2 k}$ is the lowest component of a linear combination $T^{\prime}$ of functions of the form $\frac{\cosh (2 k-5) \delta}{\cosh \delta}$. So $P$ is the lowest component of the linear combination $T T^{\prime}$. If we now fix $\Lambda^{\prime}$, then the equation for $\Lambda^{\prime \prime}$ obtained from typicality conditions has finitely many solutions. Hence, by selecting sufficiently large numbers $k$ in $T^{\prime}$ we may assume that any highest weight of the linear combination $T T^{\prime}$ satisfies the conditions for finite dimension and typicality. 
This implies that the lowest component of $\prod_{\alpha \in \tilde{R}_{\overline{1}}^{+}}\left(e^{\alpha / 2}-e^{-\alpha / 2}\right) \cdot T T^{\prime}$, equal to $P_{2}$, is the restriction of an invariant.

$\underline{\mathfrak{g}}=\mathfrak{a b}_{3}$. In this case $\mathfrak{g}_{\overline{0}}=\mathfrak{o}(7) \oplus \mathfrak{s l}(2)$ and $\mathfrak{g}_{\overline{1}}=\operatorname{spin}(7) \otimes$ id. Let $\pm \varepsilon_{1}, \pm \varepsilon_{2}, \pm \varepsilon_{3}$ be the weights of the standard $\mathfrak{o}(7)$-module and $\pm \frac{1}{2} \delta$ the weights of the identity representation of $\mathfrak{s l}(2)$. Then

$$
R_{\overline{0}}=\left\{ \pm \varepsilon_{i} ; \pm \varepsilon_{i} \pm \varepsilon_{j} ; \pm \delta\right\}, \quad R_{\overline{1}}=\left\{\frac{1}{2}\left( \pm \varepsilon_{1} \pm \varepsilon_{2} \pm \varepsilon_{3} \pm \delta\right)\right\}
$$

For the system of simple roots select

$$
\frac{1}{2}\left(\delta-\varepsilon_{1}-\varepsilon_{2}-\varepsilon_{3}\right), \varepsilon_{1}-\varepsilon_{2}, \varepsilon_{2}-\varepsilon_{3}, \varepsilon_{3} .
$$

Then

$$
\begin{aligned}
& R_{\overline{0}}^{+}=\left\{\varepsilon_{2} ; \varepsilon_{2}, \varepsilon_{3}, \varepsilon_{i} \pm \varepsilon_{j} \text { for } i<j, \delta\right\} \\
& R_{\overline{1}}^{+}=\left\{\frac{1}{2}\left(\delta \pm \varepsilon_{1} \pm \varepsilon_{2} \pm \varepsilon_{3}\right)\right\}
\end{aligned}
$$

Clearly, $\rho_{1}=2 \delta$.

The restriction of the nondegenerate invariant supersymmetric even bilinear form $\operatorname{str}(\operatorname{ad} x \cdot \operatorname{ad} y)$ onto the Cartan subalgebra is proportional to

$$
3\left(\varepsilon_{1}^{2}+\varepsilon_{2}^{2}+\varepsilon_{3}^{2}\right)-\delta^{2} .
$$

From the condition $\left(h_{\alpha}, h\right)=\alpha(h)$ for $h \in \mathfrak{h}$ we deduce for $\alpha=\frac{1}{2}\left(\varepsilon_{1}+\varepsilon_{2}+\varepsilon_{3}+\delta\right)$ that

$$
h_{\alpha}=\frac{1}{6}\left(H_{1}+H_{2}+H_{3}\right)-\frac{1}{2} H,
$$

where $H_{1}, H_{2}, H_{3}$ and $H$ is the basis of $\mathfrak{h}$ dual to $\varepsilon_{1}, \varepsilon_{2}, \varepsilon_{3}$ and $\delta$. Therefore, the condition $D_{h_{\alpha}} f \in(\alpha)$ is equivalent to the following one:

$$
\frac{\partial f}{\partial \varepsilon_{1}}+\frac{\partial f}{\partial \varepsilon_{2}}+\frac{\partial f}{\partial \varepsilon_{3}}-3 \frac{\partial f}{\partial \delta} \in\left(\delta+\varepsilon_{1}+\varepsilon_{2}+\varepsilon_{3}\right) .
$$

The other conditions of a similar type follow from $W$-invariance. Observe that $W$ is isomorphic to $\left(S_{3} \circ(\mathbb{Z} / 2)^{3}\right) \times \mathbb{Z} / 2$. Direct calculations demonstrate that $I\left(\mathfrak{h}^{*}\right)$ consists of the elements of the form

$$
P_{1}\left(\mu_{-2}, \mu_{2}\right)+\prod_{\alpha \in R_{\overline{1}}^{+}} \alpha \cdot P_{2},
$$

where the $\mu_{i}$ are the coefficients of $t^{i}$ in the power series expansion of the rational function $\frac{\prod_{\alpha \in R_{\overline{1}}}(t-\alpha)}{\prod_{\alpha \in R_{\overline{0}}}(t-\alpha)}$ and $P_{2} \in S\left(\mathfrak{h}^{*}\right)^{W}$.

It is clear that $\mu_{ \pm 2}$ are the restrictions of invariants; we prove that $\prod_{\alpha \in R_{\overline{1}}^{+}} \alpha \cdot P_{2}$ is the restriction of an invariant by the same arguments as in the last two cases above. 


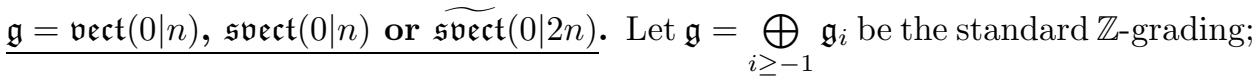
$\varepsilon_{1}, \ldots, \varepsilon_{n}$ the weights of the $\mathfrak{g}_{0}$-module $\mathfrak{g}_{-1}$. Then it is not difficult to verify that $\left[\mathfrak{g}_{\overline{1}}^{\alpha}, \mathfrak{g}_{\overline{1}}^{-\alpha}\right]=\operatorname{ker} \alpha$ for $\alpha=\varepsilon_{i}$ and the condition $D_{h_{\alpha}} f \in(\alpha)$ implies that $\left.f\right|_{\text {ker } \alpha}=$ const $=c$.

Hence, $f-c$ is divisible by $\alpha$ and by $W$-symmetry it is divisible by $\varepsilon_{1} \ldots \varepsilon_{n}$ since the factors are mutually prime. Therefore, any element from $I\left(\mathfrak{h}^{*}\right)$ is of the form

$$
c+\varepsilon_{1} \ldots \varepsilon_{n} \cdot P, \text { where } P \in S\left(\mathfrak{h}^{*}\right)^{W} .
$$

Proposition 1.4 implies that such an element is the restriction of an invariant.

$\mathfrak{g}=\mathfrak{p} \mathfrak{e}(n)$. Since $\mathfrak{g}$ possesses a compatible $\mathbb{Z}$-grading of depth 1 , there is a oneto-one correspondence between the irreducible $\mathfrak{g}$-modules and the irreducible $\mathfrak{g}_{0^{-}}$ modules. Therefore, arguments similar to the ones applied for $\mathfrak{g}=\mathfrak{g l}$ show that any of the elements from $J\left(\mathfrak{h}^{*}\right)$ (which is similarly defined) are linear combinations of supercharacters of finite dimensional representations. Now, let $P \in I\left(\mathfrak{h}^{*}\right)$. If $\pm \varepsilon_{1}, \ldots, \pm \varepsilon_{n}$ are the weights of the identity $\mathfrak{g}$-module, then

$$
R_{\overline{1}}=\left\{ \pm\left(\varepsilon_{i}+\varepsilon_{j}\right) \text { for } i \neq j,-2 \varepsilon_{i}\right\} .
$$

Let $e_{1}, \ldots, e_{n}$ be the basis of $\mathfrak{h}$ dual to $\varepsilon_{1}, \ldots, \varepsilon_{n}$. Then for $\alpha=\varepsilon_{i}+\varepsilon_{j}$ for $i \neq j$ and $h_{\alpha}=e_{i}-e_{j}$ the condition $D_{h_{\alpha}} f \in(\alpha)$ means that

$$
\frac{\partial P}{\partial \varepsilon_{i}}-\frac{\partial P}{\partial \varepsilon_{j}} \in\left(\varepsilon_{i}+\varepsilon_{j}\right)
$$

Equivalently, one can say that $P$ does not depend on $t$ after the substitution $\varepsilon_{i}=$ $-\varepsilon_{j}=t$.

The same arguments as for $\mathfrak{g}=\mathfrak{g l}(m \mid n)$ prove that if $P \in I\left(\mathfrak{h}^{*}\right)$, then

$$
f=P\left(e^{\varepsilon_{1} / 2}-e^{-\varepsilon_{1} / 2}, \ldots, e^{\varepsilon_{n} / 2}-e^{-\varepsilon_{n} / 2}\right) \in J\left(\mathfrak{h}^{*}\right)
$$

and the homogeneous component of $f$ of the least degree is equal to $P$.

$\mathfrak{g}=\mathfrak{s p e}(n), n \neq 4$. The answer is the same for all simple Lie superalgebras $\mathfrak{s p e}(n)$, but the proof is different, as we will see. The exceptional case $n=4$ is considered separately.

Let us show that the restriction map $I\left(\mathfrak{h}^{*}\right) \longrightarrow I\left(\tilde{\mathfrak{h}}^{*}\right)$, where $\mathfrak{h}$ is the Cartan subalgebra in $\mathfrak{p e}(n)$, while $\tilde{\mathfrak{h}}$ is the same in $\mathfrak{s p e}(n)$, is surjective.

In notations of the above case, observe that

$$
I\left(\mathfrak{h}^{*}\right)=\mathbb{C}\left[\Delta_{1}, \Delta_{3}, \ldots, \Delta_{2 k+1}, \ldots\right],
$$

where $\Delta_{l}=\sum_{i=1}^{n} \varepsilon_{i}^{l}$. Let $\tilde{\varepsilon}_{i}$ be the restriction of $\varepsilon_{i}$ onto $\tilde{\mathfrak{h}}$. Then

$$
I\left(\tilde{\mathfrak{h}}^{*}\right)=\mathbb{C}\left[\sigma_{1}, \sigma_{3}, \ldots, \sigma_{2 k+1}, \ldots\right],
$$

where $\sigma_{l}=\sum_{i=1}^{n-1} \tilde{\varepsilon}_{i}^{l}$.

Let $f \in I\left(\tilde{\mathfrak{h}}^{*}\right)$, then $f=F\left(\sigma_{1}, \sigma_{3}, \ldots, \sigma_{2 k+1}, \ldots\right)$. Consider the difference:

$$
f-F\left(\sigma_{1}-\sigma_{1}, \sigma_{3}-\sigma_{1}^{3}, \ldots, \sigma_{2 k+1}-\sigma_{1}^{2 k+1}, \ldots\right) .
$$

Under substitution $\sigma_{1}=0$ the difference vanishes, hence, is divisible by $\sigma_{1}$. Since $\sigma_{2 k+1}-\sigma_{1}^{2 k+1}$ is the image of an element from $I\left(\mathfrak{h}^{*}\right)$ (namely, of $\Delta_{2 k+1}$ ), we may assume that $f$ is divisible by $\sigma_{1}$, i.e., $f=\sigma_{1} g$. 
Let $\alpha=\varepsilon_{n-1}+\varepsilon_{n}$. The condition $D_{h_{\alpha}} f \in(\alpha)$ means that the restriction of $f$ onto ker $\alpha$ is invariant with respect to translations by $h_{\alpha}$. Since $\sigma_{1}\left(h_{\alpha}\right) \neq 0$, any element from $\operatorname{ker} \alpha$ can be expressed in the form

$$
h+t h_{\alpha}, \text { where } h \in \operatorname{ker} \alpha \cap \operatorname{ker} \sigma_{1} .
$$

Therefore,

$$
f\left(h+t h_{\alpha}\right)=\sigma_{1}\left(h+t h_{\alpha}\right) g\left(h+t h_{\alpha}\right)=t \sigma_{1}\left(h_{\alpha}\right) g\left(h+t h_{\alpha}\right)=f(h),
$$

hence, $f\left(h+t h_{\alpha}\right)=0$. Thus, $f$ is divisible by $\alpha$ and from $W$-symmetry and the fact that for $n \neq 4$ the linear functions $\alpha$ from $\tilde{R}_{\overline{1}}^{+}$are mutually prime we deduce that $f$ is divisible by $\prod_{\alpha \in \tilde{R}_{\overline{1}}} \alpha$, i.e.,

$$
f=\prod_{\alpha \in \tilde{R}_{\overline{1}}} \alpha \cdot \varphi, \text { where } \varphi \in S\left(\tilde{\mathfrak{h}}^{*}\right)^{W} .
$$

By Proposition 1.4 such an element is the restriction of an invariant.

$\mathfrak{g}=\mathfrak{s p e}(4)$. In this subcase the root spaces are 2-dimensional.

Let $\tilde{\varepsilon}_{i}$ be the restriction of $\varepsilon_{i} \in \mathfrak{h}^{*}$ onto $\tilde{\mathfrak{h}}$, where $\mathfrak{h}$ is the Cartan subalgebra in $\mathfrak{p e}(4)$ and $\tilde{\mathfrak{h}}$ is same in $\mathfrak{s p e}(4)$. Then $\alpha_{i}=\tilde{\varepsilon}_{1}+\tilde{\varepsilon}_{2}+\tilde{\varepsilon}_{3}-\tilde{\varepsilon}_{i}$. The direct calculations show that $I\left(\tilde{\mathfrak{h}}^{*}\right)$ consists of the elements of the form

$$
c+\alpha_{1} \alpha_{2} \alpha_{3} g+\left(\alpha_{1} \alpha_{2} \alpha_{3}\right)^{2} \varphi,
$$

where $c \in \mathbb{C}, g$ is any linear combination of the coefficients of the rational function

$$
F(t)=\prod_{i=1}^{4}\left(t+\tilde{\varepsilon}_{i}\right)^{-1}\left(t-\tilde{\varepsilon}_{i}\right) .
$$

By Proposition 1.4, $\left(\alpha_{1} \alpha_{2} \alpha_{3}\right)^{2} \varphi$ is the restriction of an invariant whereas $\alpha_{1} \alpha_{2} \alpha_{3} g$ can be expressed via supertraces of the standard $\mathfrak{s p e}(4)$-module.

The theorem is completely proved.

Corollary. Any element from $S\left(\mathfrak{h}^{*}\right)^{W}$ can be expressed in the form $\frac{P}{Q}$, where $P \in$ $I\left(\mathfrak{h}^{*}\right)$ and $Q=\prod_{\alpha \in \tilde{R}_{\overline{1}}} \alpha$.

\section{Anti-INVARIANT POLYNOMIALS}

The invariant polynomials appeared in $\S 2$ not only as elements of $S\left(\mathfrak{h}^{*}\right)^{W}$, but also as homogeneous components of supercharacters considered as formal power series. In this section, instead of supercharacters, I consider just characters and formulate (and prove) the corresponding analog of Chevalley's theorem.

Observe that in this case both the formulation and the proof are much easier.

3.1. The two g-module structures on $U(\mathfrak{g})$. It is well-known that the adjoint representation of $\mathfrak{g}$ can be uniquely extended to a representation in $S(\mathfrak{g})$ and $U(\mathfrak{g})$; moreover, the canonical symmetrization (actually, supersymmetrization) $\omega: S(\mathfrak{g}) \longrightarrow U(\mathfrak{g})$ given by the formula

$$
\omega\left(x_{1} \ldots x_{n}\right)=\frac{1}{n !} \sum_{\sigma \in S_{n}} c(p(x), \sigma) x_{\sigma(1)} \ldots x_{\sigma(n)},
$$

where $c(p(x), \sigma)$ is defined in (0.5.2), is an isomorphism of $\mathfrak{g}$-modules. 
Denote: $U^{n}(\mathfrak{g})=\omega\left(S^{n}(\mathfrak{g})\right)$; we get a decomposition of $U(\mathfrak{g})$ into the direct sum of $\mathfrak{g}$-modules.

As for the Lie algebra case, the space $U(\mathfrak{g})^{*}$ can be endowed with the superalgebra structure by means of the coalgebra structure $\left(m^{*}: \mathfrak{g} \longrightarrow U(\mathfrak{g}) \otimes U(\mathfrak{g})\right.$ given by $\left.m^{*}(x)=x \otimes 1+1 \otimes x\right)$.

We similarly prove that $U(\mathfrak{g})^{*}$ is isomorphic (as a superspace) to the supercommutative superalgebra of formal power series in $\operatorname{dim} \mathfrak{g}_{\overline{0}}$ even indeterminates and $\operatorname{dim} \mathfrak{g}_{\overline{1}}$ odd ones.

The corresponding isomorphism is given as follows. Let $e_{1}, \ldots, e_{n}$ be a $\mathbb{C}$-basis of $\mathfrak{g}$ and $x_{1}, \ldots, x_{n}$ the corresponding indeterminates of the same parity. Set

$$
e_{\nu}=\frac{e_{1}^{\nu_{1}}}{\nu_{1} !} \ldots \frac{e_{n}^{\nu_{n}}}{\nu_{n} !}
$$

where $\nu_{1}, \ldots, \nu_{n}$ take values in $\mathbb{Z}_{+}$for even basis vectors and are equal to either 0 or 1 for the odd ones. Then

$$
U(\mathfrak{g})^{*} \ni f \longrightarrow S_{f}=\sum f\left(e_{\nu}\right) x_{n}^{\nu_{n}} \ldots x_{1}^{\nu_{1}} .
$$

(Notice the inverse order of indeterminates.)

The homogeneous component of degree $k$ of the functional $f$ is a functional $f_{k}$ such that

$$
\left.f_{k}\right|_{U^{k}(\mathfrak{g})}=\left.f\right|_{U^{k}(\mathfrak{g})} \text { and }\left.f_{k}\right|_{U^{l}(\mathfrak{g})}=0 \text { for } l \neq k .
$$

This splitting of $f$ into homogeneous components helps us to embed $S\left(\mathfrak{g}^{*}\right)$ into $U(\mathfrak{g})^{*}$ by considering $S\left(\mathfrak{g}^{*}\right)$ as series whose homogeneous components of sufficiently high degree vanish.

Now, let us endow $U(\mathfrak{g})$ with a $\mathfrak{g}$-module structure by means of Lemma 1.2 and $U(\mathfrak{g})^{*}$ with the structure of a dual $\mathfrak{g}$-module.

3.2. Proposition. Consider symmetrization $\omega: S\left(\mathfrak{g}_{\overline{0}}\right) \longrightarrow U\left(\mathfrak{g}_{\overline{0}}\right)$ and extend it to a $\mathfrak{g}$-module homomorphism $\tilde{\omega}: S(\mathfrak{g}) \longrightarrow U(\mathfrak{g})$, where $S(\mathfrak{g})$ is considered as $\operatorname{Ind}_{\mathfrak{g}_{\overline{0}}}^{\mathfrak{g}} S\left(\mathfrak{g}_{\overline{0}}\right)$ and $U(\mathfrak{g})$ is considered as the $\mathfrak{g}$-module with respect to the structure (0.5.3). Then

(i) $\tilde{\omega}$ is a $\mathfrak{g}$-module isomorphism; $V$;

(ii) $\operatorname{tr}_{V}$ is a $\mathfrak{g}$-invariant element from $U(\mathfrak{g})^{*}$ for any finite dimensional $\mathfrak{g}$-module

(iii) let $\tilde{U}^{k}(\mathfrak{g})=\tilde{\omega}\left(\tilde{S}^{k}(\mathfrak{g})\right)$, where $\tilde{S}^{k}(\mathfrak{g})$ is the $\mathfrak{g}$-submodule generated by $S^{k}\left(\mathfrak{g}_{\overline{0}}\right)$. Then

$$
U(\mathfrak{g})=\bigoplus_{k \geq 0} \tilde{U}^{k}(\mathfrak{g})
$$

and each of the homogeneous (with respect to (3.1) components of an invariant element is an invariant itself.

Proof. (i) It suffices to show that the image of any basis of $S(\mathfrak{g})$ under $\tilde{\omega}$ is a basis of $\operatorname{gr} U(\mathfrak{g})$; but this is obvious,

(ii) follows from Lemma 1.2;

(iii) follows from the fact that $\tilde{U}^{k}(\mathfrak{g})$ is a $\mathfrak{g}$-submodule.

3.3. Theorem. Let $\mathfrak{g}$ be one of the Lie superalgebras (0.1), section 0.4 , let $\mathfrak{h}$ be its Cartan subalgebra, $W$ its Weyl group (0.3). Then the restriction homomorphism onto $\mathfrak{h}$ induces an isomorphism $S\left(\mathfrak{g}^{*}\right)^{\mathfrak{g}} \longrightarrow S\left(\mathfrak{h}^{*}\right)^{W}$. 
Proof. The embedding $\mathfrak{h} \longrightarrow \mathfrak{g}$ induces the restriction homomorphism $U(\mathfrak{g})^{*} \longrightarrow$ $S\left(\mathfrak{h}^{*}\right)$. Since $U(\mathfrak{g})=\operatorname{Ind}_{\mathfrak{g}_{\overline{0}}}^{\mathfrak{g}} U\left(\mathfrak{g}_{\overline{0}}\right)$, there exists a bijection between the set of invariant elements from $U(\mathfrak{g})^{*}$ and the $\mathfrak{g}_{0}$-invariant elements from $U\left(\mathfrak{g}_{\overline{0}}\right)^{*}$.

Therefore, we may assume that $\mathfrak{g}=\mathfrak{g}_{\overline{0}}$. If $\mathfrak{g}_{\overline{0}}$ is reductive, the theorem is obtained as a corollary of Chevalley's theorem for the Lie algebras. If $\mathfrak{g}_{\overline{0}}$ is not reductive, it suffices to demonstrate that one can replace $\mathfrak{g}_{\overline{0}}$ with $\mathfrak{g}_{0}$ for the standard $\mathbb{Z}$-grading when $\mathfrak{g}_{0}$ is reductive and apply Chevalley's theorem for the Lie algebras again.

\section{Appendix. Certain constructions with the point functor}

The point functor is well-known in algebraic geometry since at least 1953 [W]. The advertising of ringed spaces with nilpotents in the structure sheaf that followed the discovery of supersymmetries caused many mathematicians and physicists to realize the usefulness of the language of points. F. A. Berezin was the first who applied the point functor to study Lie superalgebras. Here we present some of his results and their generalizations.

All superalgebras and modules are supposed to be finite dimensional over $\mathbb{C}$.

Thus, let $\mathfrak{g}$ be a Lie superalgebra, $V$ a $\mathfrak{g}$-module, $\Lambda$ the Grassmann superalgebra over $\mathbb{C}$ generated by $q$ indeterminates. Define $\varphi: \Lambda \otimes V^{*} \longrightarrow \operatorname{Hom}_{\Lambda}(\Lambda \otimes V, \Lambda)$ by setting

$$
\varphi(\xi \otimes \alpha)(\eta \otimes v)=(-1)^{p(\alpha)(\eta)} \xi \eta \alpha(v), \text { for any } \xi, \eta \in \Lambda, \alpha \in V^{*} .
$$

Extend the ground field to $\Lambda$ and consider $\Lambda \otimes V^{*}$ and $\operatorname{Hom}_{\Lambda}(\Lambda \otimes V, \Lambda)$ as $\Lambda \otimes \mathfrak{g}$ modules.

A.1. Lemma. $\varphi$ is a $\Lambda \otimes \mathfrak{g}$-module isomorphism.

Proof. Since $V$ is finite dimensional, $\varphi$ is a vector space isomorphism over $\Lambda$; besides, it is obvious that $\varphi$ is a $\Lambda$-module homomorphism. Now take

$$
\xi_{1}, \xi_{2}, \xi_{3} \in \Lambda, \quad \alpha \in V^{*}, \quad v \in V, \quad x \in \mathfrak{g} .
$$

It is an easy exercise to prove that

$$
\left[\left(\xi_{1} \otimes x\right) \varphi\left(\xi_{2} \otimes \alpha\right)\right]\left(\xi_{3} \otimes v\right)=\varphi\left(\xi_{1} \otimes x\left(\xi_{2} \otimes \alpha\right)\right)\left(\xi_{3} \otimes v\right) .
$$

Consider the composition of maps

$$
V^{*} \stackrel{\varphi_{1}}{\longrightarrow} \Lambda \otimes V^{*} \stackrel{\varphi}{\longrightarrow} \operatorname{Hom}_{\Lambda}(\Lambda \otimes V, \Lambda) \stackrel{\varphi_{2}}{\longrightarrow} S_{\Lambda}\left(\operatorname{Hom}_{\Lambda}(\Lambda \otimes V, \Lambda)\right),
$$

where $\varphi_{1}(\alpha)=1 \otimes \alpha$ and $\varphi_{2}$ is a canonical embedding of a module in its symmetric algebra. The $\mathbb{C}$-module homomorphism $\varphi_{2} \circ \varphi \circ \varphi_{1}$ induces the algebra homomorphism

$$
S\left(V^{*}\right)=S_{\mathbb{C}}\left(V^{*}\right) \longrightarrow S_{\Lambda}\left(\operatorname{Hom}_{\Lambda}(\Lambda \otimes V, \Lambda)\right)
$$

and, since the latter algebra is a $\Lambda$-module, we get an algebra homomorphism

$$
\Lambda \otimes S\left(V^{*}\right) \stackrel{\psi}{\longrightarrow} S_{\Lambda}\left(\operatorname{Hom}_{\Lambda}(\Lambda \otimes V, \Lambda)\right) .
$$

Besides, both algebras possess a natural $\Lambda \otimes \mathfrak{g}$-module structure.

A.2. Lemma. $\psi$ is a $\Lambda \otimes \mathfrak{g}$-module and $\Lambda \otimes \mathfrak{g}$-algebras are isomorphisms. 
Proof. Let us construct the inverse homomorphism. Consider the composition

$$
\operatorname{Hom}_{\Lambda}(\Lambda \otimes V, \Lambda) \stackrel{\varphi^{-1}}{\longrightarrow} \Lambda \otimes V^{*} \longrightarrow \Lambda \otimes S\left(V^{*}\right) .
$$

Since this composition is a $\Lambda$-module homomorphism, it induces the homomorphism

$$
\tilde{\psi}: S_{\Lambda}\left(\operatorname{Hom}_{\Lambda}(\Lambda \otimes V, \Lambda)\right) \longrightarrow \Lambda \otimes S\left(V^{*}\right) .
$$

It is not difficult to verify that

$$
\left.\psi \circ \tilde{\psi}\right|_{\operatorname{Hom}_{\Lambda}(\Lambda \otimes V, \Lambda)}=\mathrm{id} ;\left.\quad \tilde{\psi} \circ \psi\right|_{\Lambda \otimes S\left(V^{*}\right)}=\mathrm{id} ;
$$

hence, $\psi$ is an isomorphism and $\tilde{\psi}$ is its inverse. The following proposition shows that $\psi$ is a $\Lambda \otimes \mathfrak{g}$-module isomorphism and completes the proof of Lemma A.2.

A.3. Proposition. Let $A, B$ be $\Lambda$-superalgebras, $\mathfrak{g}$ a Lie superalgebra over $\Lambda$ acting by differentiations on $A$ and $B$. Let $M \subset A, N \subset B$ be $\Lambda$-submodules which are at the same time $\mathfrak{g}$-modules generating $A$ and $B$, respectively, $f: A \longrightarrow B$ an algebra homomorphism such that $f(M) \subset N$ and $\left.f\right|_{M}$ is a $\mathfrak{g}$-module homomorphism. Then $f$ is a $\mathfrak{g}$-module homomorphism.

Proof. Let $a \in A$. We may assume that $a=a_{1} \ldots a_{n}$, where the $a_{i} \in M$. Then for $x \in \mathfrak{g}$ we have

$$
\begin{gathered}
f\left(x\left(a_{1} \ldots a_{n}\right)\right)=f\left(\sum \pm a_{1} \ldots x a_{i} \ldots a_{n}\right)=\sum \pm f\left(a_{1}\right) \ldots f\left(x a_{i}\right) \ldots f\left(a_{n}\right) \\
\quad=\sum \pm f\left(a_{1}\right) \ldots x f\left(a_{i}\right) \ldots f\left(a_{n}\right)=x\left[f\left(a_{1}\right) \ldots f\left(a_{n}\right)\right]=x f\left(a_{1} \ldots a_{n}\right) .
\end{gathered}
$$

This proves the proposition and completes the proof of Lemma A.2.

Now, let $\mathfrak{h}$ be a Lie superalgebra over $\Lambda$ and $U$ be a $\Lambda$ and $\mathfrak{h}$-module. Consider $U_{\overline{0}}$ as a $\mathbb{C}$-module. Then, clearly, the natural embedding $U_{\overline{0}} \longrightarrow U$ is extendable to a $\Lambda$-module homomorphism $\varphi: \Lambda \otimes U_{\overline{0}} \longrightarrow U$.

A.4. Lemma. The homomorphism $\varphi$ is an $\mathfrak{h}_{\overline{0}}$-module homomorphism.

Proof. Let $x \in \mathfrak{h}_{\overline{0}}, \xi \in \Lambda$ and $u \in U_{\overline{0}}$. Then

$$
\varphi(x(\xi \otimes u))=\varphi(\xi \otimes x u)=\xi x u,
$$

$x \varphi(\xi \otimes u)=x \xi u=$ (by definition of a module over a superalgebra) $\xi x u$.

Thus, the adjoint map

$$
\operatorname{Hom}_{\Lambda}(U, \Lambda) \longrightarrow \operatorname{Hom}_{\Lambda}\left(\Lambda \otimes U_{\overline{0}}, \Lambda\right)
$$

is also an $\mathfrak{h}_{\overline{0}}$-module homomorphism, therefore, by Proposition A.3 the algebra homomorphism

$$
S_{\Lambda}\left(\operatorname{Hom}_{\Lambda}(U, \Lambda)\right) \longrightarrow S_{\Lambda}\left(\operatorname{Hom}_{\Lambda}\left(\Lambda \otimes U_{\overline{0}}, \Lambda\right)\right)
$$

induced by this map is at the same time a $\mathfrak{h}_{\overline{0}}$-module morphism. Besides, by Lemma A.2 the algebra $S_{\Lambda}\left(\operatorname{Hom}_{\Lambda}\left(\Lambda \otimes U_{\overline{0}}, \Lambda\right)\right)$ is isomorphic as a $\Lambda \otimes \mathfrak{h}_{\overline{0}}$-module and as an algebra to $\Lambda \otimes S\left(U_{\overline{0}}^{*}\right)$. In particular, they are isomorphic as $\mathfrak{h}_{\overline{0}}$-modules.

Denote by $\theta$ the composition of the homomorphisms

$$
S\left(V^{*}\right) \longrightarrow \Lambda \otimes S\left(V^{*}\right) \longrightarrow S_{\Lambda}\left(\operatorname{Hom}_{\Lambda}(\Lambda \otimes V, \Lambda)\right) \longrightarrow S_{\Lambda}\left(\operatorname{Hom}_{\Lambda}\left(\Lambda \otimes U_{\overline{0}}, \Lambda\right)\right),
$$

where $U_{\overline{0}}=(\Lambda \otimes V)_{\overline{0}}=V_{\Lambda}$.

A.5. Proposition. If $q>\operatorname{dim} V_{\overline{1}}$ and $\xi \in \Lambda, p(\xi)=\overline{1}$, then the restriction of $\theta$ onto $\mathbb{C}[\xi] \otimes S\left(V^{*}\right)$ is injective. 
Proof. If $u \in V_{\Lambda}$, then there is defined a linear form $L_{u}: \operatorname{Hom}_{\Lambda}\left(\Lambda \otimes V_{\Lambda}, \Lambda\right) \longrightarrow \Lambda$ by the formulas $L_{u}(l)=l(1 \otimes u)$ and $L_{u}(\xi l)=\xi l(1 \otimes u)=\xi L_{u}(l)$.

Therefore, $L_{u}$ is a $\Lambda$-module homomorphism, hence, it is uniquely extendable to a homomorphism

$$
\varphi_{u}: \mathfrak{a}=S_{\Lambda}\left(\operatorname{Hom}_{\Lambda}\left(\Lambda \otimes V_{\Lambda}, \Lambda\right)\right) \longrightarrow \Lambda .
$$

Consider the elements of $\mathfrak{a}$ as functions on $V_{\Lambda}$ setting $f(u)=\varphi_{u}(f)$ for $f \in \mathfrak{a}$ and $u \in V_{\Lambda}$. If $f \in \Lambda \otimes S\left(V^{*}\right)$, then set $f(u)=\varphi_{u} \circ \theta(f)$. For $\alpha \in V^{*}, \xi \in \Lambda$ we have

$$
(\xi \otimes \alpha)(u)=\varphi_{u} \circ \theta(\xi \otimes \alpha)=L_{u} \circ \theta(\xi \otimes \alpha)=\theta(\xi \otimes \alpha)(1 \otimes u) .
$$

If $\left\{e_{i}\right\}_{i \in I}$ is a basis in $V$ and $u=\sum \lambda_{i} \otimes e_{i}$, then

$$
(\xi \otimes \alpha)(u)=\sum(-1)^{p(\alpha) p\left(e_{i}\right)} \xi \lambda_{i} \alpha\left(e_{i}\right) .
$$

On the other hand, the algebra $\mathbb{C}[\xi] \otimes S\left(V^{*}\right)$ is identified with the free supercommutative superalgebra generated by the $e_{i}^{*}$ and $\xi$.

Let us assume that $p\left(e_{i}^{*}\right)=0$ for $i \leq n$ and $p\left(e_{i}^{*}\right)=1$ for $i>n$. If $f \in$ $\mathbb{C}[\xi] \otimes S\left(V^{*}\right)$, then

$$
f=f_{0}+\xi f_{1}, f_{j}=\sum f_{j i_{1} \ldots i_{k}} e_{i_{1}}^{*} \ldots e_{i_{k}}^{*}, \text { where } j=0,1 \text { and } f_{j i_{1} \ldots i_{k}} \in S\left(V_{\overline{0}}^{*}\right) .
$$

By (1) we have

$$
\begin{gathered}
f(u)=f_{0}(u)+\xi f_{1}(u) \\
=\sum f_{0 i_{1} \ldots i_{k}}(u) e_{i_{1}}^{*}(u) \ldots e_{i_{k}}^{*}(u)+\sum f_{1 i_{1} \ldots i_{k}}(u) e_{i_{1}}^{*}(u) \ldots e_{i_{k}}^{*}(u) .
\end{gathered}
$$

Set $\lambda_{i}=a_{i}$ for $i \leq n$ and $\lambda_{i}=\xi_{i-n}$ for $i>n$. Then since $q>\operatorname{dim} V_{\overline{1}}$, we may assume that the family $\left\{\xi_{i}\right\}_{i \in I}$ freely generates $S\left(V_{\overline{1}}^{*}\right)$ and

$$
f(u)=\sum(-1)^{k} f_{i_{1} \ldots i_{k}}\left(a_{1} \ldots a_{n}\right) \xi_{i_{1}-n \ldots i_{k}-n} .
$$

If $\theta(f)=0$, then $f(u)=\varphi_{u} \circ \theta(f)$ for any $u \in V_{\Lambda}$. It follows from (2) that $f_{i_{1} \ldots i_{k}}(a)=0$ for any $a \in \mathbb{C}^{n}$. But since $\mathbb{C}$ is algebraically closed, it follows (with Prop. 5.3.1 from [Bu3]) that $f_{i_{1} \ldots i_{k}}=0$; hence, $f=0$.

A.6. Lemma. Let $q>\operatorname{dim} V_{\overline{1}}$. Then $f \in S\left(V^{*}\right)$ is a $\mathfrak{g}$-invariant if and only if $\theta(f) \in \Lambda \otimes S\left(V_{\Lambda}^{*}\right)$ is $\mathfrak{g}_{\Lambda}$-invariant.

Proof. Consider the factorization of $\theta$ :

$$
\begin{gathered}
S\left(V^{*}\right) \stackrel{i_{1}}{\longrightarrow} \Lambda \otimes S\left(V^{*}\right) \stackrel{i_{2}}{\longrightarrow} \\
S_{\Lambda}\left(\operatorname{Hom}_{\Lambda}(\Lambda \otimes V, \Lambda)\right) \stackrel{i_{3}}{\longrightarrow} S_{\Lambda}\left(\operatorname{Hom}_{\Lambda}\left(\Lambda \otimes V_{\Lambda}, \Lambda\right)\right) \stackrel{i_{4}}{\longrightarrow} \Lambda \otimes S\left(V_{\Lambda}^{*}\right) .
\end{gathered}
$$

Let $f \in S\left(V^{*}\right)^{\mathfrak{g}}$, then

$$
(\xi \otimes x)\left(i_{1}(f)\right)=(\xi \otimes x)(1 \otimes f)=\xi \otimes x f=0 \quad \text { for } \xi \in \Lambda, x \in \mathfrak{g} .
$$

Conversely, let $y i_{1}(f)=0$ for any $y \in \mathfrak{g}_{\Lambda}$. Then

$$
0=(\xi \otimes x)(1 \otimes f)=\xi \otimes x f .
$$

If $p(y)=\overline{1}$, then let $p(\xi)=\overline{1}$. Therefore, $\xi \otimes x f=0$ and $y f=0$.

Thus, conditions

$$
f \in S\left(V^{*}\right)^{\mathfrak{g}} \longleftrightarrow i_{1}(f) \in\left(\Lambda \otimes S\left(V^{*}\right)\right)_{\Lambda}^{\mathfrak{g}} .
$$

Since $i_{2}, i_{3}, i_{4}$ are $\mathfrak{g}_{\Lambda}$-module homomorphisms, the above implies that if $f$ is a $\mathfrak{g}$-invariant, then $\theta(f)=i_{4} \circ i_{3} \circ i_{2} \circ i_{1}(f)$ is also a $\mathfrak{g}_{\Lambda}$-invariant. 
Conversely, let $\theta(f)$ be a $\mathfrak{g}_{\Lambda}$-invariant. Let $x \in \mathfrak{g}_{\overline{0}}$. Then

$$
\theta(1 \otimes x f)=\theta((1 \otimes x)(1 \otimes f))=(1 \otimes x) \theta(f)=0 .
$$

By Proposition A.5 $1 \otimes x f=0$ and $x f=0$. Let $x \in \mathfrak{g}_{\overline{1}}, \xi \in \Lambda, p(\xi)=\overline{1}$. Then $\theta(\xi \otimes x f)=(\xi \otimes x) \theta(1 \otimes f)=0$ and again by Proposition A.5 $\xi \otimes x f=0$; hence $x f=0$ and therefore, $f \in S\left(V^{*}\right)^{\mathfrak{g}} g$.

A.7. Remark. The point of the above lemmas and propositions is that while seeking invariant polynomials on $V$ we may consider them as functions on $V_{\Lambda}$ invariant with respect to the Lie algebra $\mathfrak{g}_{\Lambda}$. It makes it possible to apply the theory of usual Lie groups and Lie algebras and their representations.

A.8. Proposition. Let $A$ be a commutative finitely generated algebra over $\mathbb{C}$ without nilpotents, $\mathfrak{a}=A \otimes \Lambda(p)$. Let $q \geq p$ and $f \in \mathfrak{a}$ be such that $\varphi(f)=0$ for any $\varphi: \mathfrak{a} \longrightarrow \Lambda(q)$. Then $f=0$.

Proof. Let $\psi: A \longrightarrow \mathbb{C}$ be an arbitrary homomorphism. Let us extend $\psi$ to a homomorphism $\varphi: \mathfrak{a} \longrightarrow \Lambda(q)$ setting $\varphi=\psi \otimes 1$. If $\xi_{1}, \ldots, \xi_{p}$ are generators of $\Lambda(p), f \in \mathfrak{a}$ and $f=\sum f_{i_{1} \ldots i_{k}} \xi_{i_{1}} \ldots \xi_{i_{k}}$, then the condition $\varphi(f)=0$ yields $\psi\left(f_{i_{1} \ldots i_{k}}\right)=0$ and, since $\psi$ is arbitrary, then Proposition 5.3.1 in [Bu3] shows that $f_{i_{1} \ldots i_{k}}=0$; hence, $f=0$.

\section{REFERENCES}

[B] Bernstein J., Finite dimensional representations of semisimple Lie algebras. (Verma module approach). In: Leites D. (ed.) Seminar on Supermanifolds, Reports of Stockholm University, n. 10, 1987-92.

[BL1] Bernstein J., Leites D., A formula for the characters of the irreducible finite-dimensional representations of Lie superalgebras of series $\mathfrak{g l}$ and $\mathfrak{s l}$. (Russian) C. R. Acad. Bulgare Sci. 33, no. 8, 1980, 1049-1051. MR 82j:17020a

[BL2] Bernstein J., Leites D., The superalgebra $Q(n)$, the odd trace and the odd determinant, C. R. Acad. Bulgare Sci. v. 35, n.3, 1982, 285-286. MR 84c:17003

[Be1] Berezin F., Representations of the supergroup $U(p, q)$. Funkcional. Anal. i Prilozhen. 10 n. 3, 1976, 70-71. (in Russian) MR 54:10495

[Be2] Berezin F., Laplace-Cazimir operators on Lie supergroups. The general theory. Preprints ITEPh 77, Moscow, ITEPh, 1977; Berezin F. Introduction to superanalysis. Edited and with a foreword by A. A. Kirillov. With an appendix by V. I. Ogievetsky. Translated from the Russian by J. Niederle and R. Kotecký. Translation edited by Dimitri Leites. Mathematical Physics and Applied Mathematics, 9. D. Reidel Publishing Co., DordrechtBoston, MA, 1987.

[Bu1] Bourbaki N., Groupes et algébres de Lie. Ch. VII-VIII, Hermann, Paris, 1978.

[Bu2] Bourbaki N., Lie groups and Lie algebras. Chapters 1-3. Translated from the French. Reprint of the 1975 edition. Elements of Mathematics. Springer-Verlag, Berlin-New York, 1989. MR 89k:17001

[Bu3] Bourbaki N., Commutative algebra. Chapters 1-7. Translated from the French. Reprint of the 1972 edition. Elements of Mathematics. Springer-Verlag, Berlin-New York, 1989. MR 90a:13001

[E] Egorov G., How to superize $\mathfrak{g l}(\infty)$. In: Mickelsson J. et. al. (eds.) Topological and geometrical methods in field theory, World Scientific, 1992, 135-146. MR 94c:17007

[K1] Kac V.G., Lie superalgebras. Adv. Math., v. 26, 1977, 8-96. MR 58:5803

[K2] Kac V.G., Characters of typical representations of classical Lie superalgebras. Commun. Alg. v. 5, 1977, 889-897. MR 56:3075

[K3] Kac V.G., Laplace operators of infinite-dimensional Lie algebras and theta functions. Proc. Nat. Acad. Sci. U.S.A. 81, no. 2, 1984, Phys. Sci., 645-647. MR 85j:17025

[L1] Leites D., A formula for the characters of the irreducible finite-dimensional representations of Lie superalgebras of series C. (Russian) C. R. Acad. Bulgare Sci. 33, no. 8, 1980, 10531055. MR 82j:17020b 
[L2] Leites D., Lie superalgebras. In: Current problems in mathematics, Vol. 25, Itogi Nauki i Tekhniki, Akad. Nauk SSSR, Vsesoyuz. Inst. Nauchn. i Tekhn. Inform., Moscow, 1984, 3-49 (in Russian; the English translation: JOSMAR, v. 30 (6), 1985, 2481-2512). MR 86f: 17019

[LSS] Leites D., Saveliev M. V., Serganova V. V., Embeddings of $\mathfrak{o s p}(N \mid 2)$ and completely integrable systems. In: Dodonov V., Man'ko V. (eds.) Proceedings of International seminar group-theoretical methods in physics, Yurmala, May 1985. Nauka, Moscow, 1986; 377394 (an enlarged version in English is published by VNU Sci Press, 1986, 255-297). MR 89a:58052

[M] Manin Yu. (ed.), Current problems in mathematics. Newest results, Vol. 32, Itogi Nauki i Tekhniki, Akad. Nauk SSSR, Vsesoyuz. Inst. Nauchn. i Tekhn. Inform., Moscow, 1988, $3-70$.

[OO] Okunkov A., Olshansky G., Shifted Schur functions. (Russian) Algebra i Analiz 9 (1997), no. 2, 73-146; English translation in St. Petersburg Math. J. 9 (1998), no. 2, 239-300; id., Shifted Schur functions. II. The binomial formula for characters of classical groups and its applications. Kirillov's seminar on representation theory, 245-271, Amer. Math. Soc. Transl. Ser. 2, 181, Amer. Math. Soc., Providence, RI, 1998. MR 99f:05118; CMP 98:12

[OV] Onishchik A., Vinberg E., Lie groups and Algebraic Groups, Springer, 1987.

[Pe1] Penkov I., Characters of strongly generic irreducible Lie superalgebra representations. Preprint ESI 341, Vienna, 1996; Internat. J. Math. 9, 1998, no. 3, 331-366.

[Pe2] Penkov I., Generic representations of classical Lie superalgebras and their localization. Monatsh. Math. 118, 1994, no. 3-4, 267-313. MR 95k:17011

[PS1] Penkov I., Serganova V., Generic irreducible representations of finite dimensional Lie superalgebras. Internat. J. Math. 5, 1994, 389-419. MR 95c:17015

[PS2] Penkov I., Serganova V., Representations of classical Lie superalgebras of type I. Indag. Math. (N.S.) 3, no. 4, 1992, 419-466. MR 93k:17006

[P] Pragacz P. Algebro-geometric applications of Schur $S$ - and $Q$-polynomials. Lecture Notes Math., v. 1478, 1991, 130-191. MR 93h:05170

[S1] Sergeev A., Laplace operators and Lie superalgebra representations. Ph.D. thesis, Moscow University, 1985. In: Leites D. (ed.) Seminar on Supermanifolds, Reports of Stockholm University, n. 32/1988-15, 44-95.

[S2] Sergeev A., Invariant polynomial functions on Lie superalgebras (Russian) C.R. Acad. Bulgare Sci., 35, n.5, 1982, 573-576. MR 84h:17015

[S3] Sergeev A., The centre of enveloping algebra for Lie superalgebra $Q(n, C)$. Lett. Math. Phys. 7, no. 3, 1983, 177-179. MR 85i:17004

[Sh] Shander V., Orbits and invariants of the supergroup $\mathrm{GQ}_{n}$. (Russian) Funktsional. Anal. i Prilozhen. 26, no. 1, 1992, 69-71; translation in Functional Anal. Appl. 26 no. 1, 1992, 55-56; for details see math.RT/9810112. MR 93j:17006

[Sch] Scheunert M., Invariant supersymmetric multilinear forms and the Casimir elements of P-type Lie superalgebras. J. Math. Phys. 28, no. 5, 1987, 1180-1191. MR 89b:17008

[W] Weil A., Théorie des points proches sur les variétés différentiables. (French) Géométrie différentielle. Colloques Internationaux du Centre National de la Recherche Scientifique, Strasbourg, 1953, pp. 111-117. Centre National de la Recherche Scientifique, Paris, 1953. MR 15:828f

On leave of absence from the Balakovo Institute of Technique of Technology and Control, Branch of Saratov State Technical University, Russia

Correspondence: c/o D. Leites, Department of Mathematics, University of Stockholm, Roslagsv. 101, Kräftriket hus 6, S-106 91, Stockholm, Sweden

E-mail address: mleites@matematik.su.se 